\title{
LONG-TIME STABILITY AND CONVERGENCE OF THE UNIAXIAL PERFECTLY MATCHED LAYER METHOD FOR TIME-DOMAIN ACOUSTIC SCATTERING PROBLEMS
}

\author{
ZHIMING CHEN* AND XINMING WU ${ }^{\dagger}$
}

\begin{abstract}
The uniaxial perfectly matched layer (PML) method uses rectangular domain to define the PML problem and thus provides greater flexibility and efficiency in dealing with problems involving anisotropic scatterers. In this paper we first derive the uniaxial PML method for solving the time-domain scattering problem based on the Laplace transform and the complex coordinate stretching in the frequency domain. We prove the long-time stability of the initial-boundary value problem of the uniaxial PML system for piecewise constant medium property and show the exponential convergence of the time-domain uniaxial PML method. Our analysis shows that for fixed PML absorbing medium property, any error of the time-domain PML method can be achieved by enlarging the thickness of the PML layer as $\ln T$ for large $T>0$. Numerical experiments are included to illustrate the efficiency of the PML method.
\end{abstract}

Key words. Uniaxial perfectly matched layer, time-domain scattering, convergence, stability.

1. Introduction. We propose and study a uniaxial perfectly matched layer (PML) method for solving the acoustic scattering problem with the sound-hard boundary condition on the obstacle:

$$
\begin{aligned}
& \frac{\partial u}{\partial t}=-\operatorname{div} \mathbf{p}+f(x, t), \quad \frac{\partial \mathbf{p}}{\partial t}=-\nabla u \quad \text { in }\left(\mathbb{R}^{2} \backslash \bar{D}\right) \times(0, T), \\
& \mathbf{p} \cdot \mathbf{n}_{D}=0 \quad \text { on } \Gamma_{D} \times(0, T), \\
& \sqrt{r}(u-\mathbf{p} \cdot \hat{x}) \rightarrow 0, \quad \text { as } r=|x| \rightarrow \infty, \quad \text { a.e. } t \in(0, T), \\
& \left.u\right|_{t=0}=u_{0},\left.\quad \mathbf{p}\right|_{t=0}=\mathbf{p}_{0} .
\end{aligned}
$$

Here $u$ is the pressure and $\mathbf{p}$ is the velocity field of the wave. $D \subset \mathbb{R}^{2}$ is a bounded domain with Lipschitz boundary $\Gamma_{D}$ and $\mathbf{n}_{D}$ is the unit outer normal to $\Gamma_{D} \cdot f, u_{0}, \mathbf{p}_{0}$ are assumed to be supported in the circle $B_{R}=\left\{x \in \mathbb{R}^{2}:|x|<R\right\}$ for some $R>0$. (1.3) is the radiation condition which corresponds to the well-known Sommerfeld radiation condition in the frequency domain. We remark that the results in this paper can be easily extended to solve scattering problems with other boundary conditions such as the sound-soft or the impedance boundary conditions on $\Gamma_{D}$.

Since the work of Bérenger [5] which proposed a PML technique for solving Maxwell equations, various constructions of PML absorbing layers have been proposed and studied in the literature (cf. e.g. Turkel and Yefet [27], Teixeira and Chew [26] for the reviews). The basic idea of the PML technique is to surround the computational domain by a layer of finite thickness with specially designed model medium that absorbs all the waves that propagate from inside the computational domain.

The initial Bérenger's PML method is based on the splitting of the electromagnectic fields, which is later proved by Abarbanel and Gottlieb [1] that it is only weakly well-posed and thus may suffer instability in practical applications. The other

*LSEC, Institute of Computational Mathematics, Academy of Mathematics and System Sciences, Chinese Academy of Sciences, Beijing 100190, People's Republic of China. This author was supported in part by National Basic Research Project under the grant 2011CB309700 and China NSF under the grant 11021101. (zmchen@lsec.cc.ac.cn).

${ }^{\dagger}$ Laboratory of Mathematics for Nonlinear Science, School of Mathematical Sciences, Fudan University, Shanghai 200433, People's Republic of China. This author was supported by China NSF under the grant 11026036. (wuxinming@fudan.edu.cn). 
so-called "unsplit-field" PML formulations are strongly well-posed, including the uniaxial PML method developed in Sacks et al [25], Zhao-Cangellaris [29], and Gedney [16] for the Maxwell equations and a second order PML formulation in Grote and Sim [17] for wave equations. In curvilinear coordinates, the split-field PML method is introduced in Collino and Monk [13] and the unsplit-field PML methods are introduced in Petropoulous [24] and [26] for Maxwell equations.

Although the PML approach has been proved very successful in the practical applications, there are few mathematical results on the convergence of the PML methods. In the frequency domain, the convergence is studied in Lassas and Somersalo [21], Hohage et al [19] for the acoustic scattering problems with the circular layers and in Chen and $\mathrm{Wu}$ [10], Kim and Pasciak [20], Chen and Zheng [11] with the uniaxial PML layers. It is proved in $[21,19,10,11]$ that the PML solution converges exponentially to the solution of the original scattering problem as the thickness of the PML layer tends to infinite. In Chen and $\mathrm{Wu}$ [9], Chen and Liu [8], an adaptive PML technique is proposed and studied in which a posteriori error estimate is used to determine the PML parameters. For the time-domain PML method, the planar PML method in one space direction is considered in Hagstrom [18] for the wave equation. In de Hoop et al [14], Diaz and Joly [15], the PML system with point source is analyzed based on the Cagniard - de Hoop method. In Chen [7], the convergence of the time-domain PML method with circular layer is proved by using the exponential decay esitmate of the modified Bessel functions.

The long-time stability of the PML method is also a much studied topic in the literature (see e.g. Bécache and Joly [3], Bécache et al [4], Appelö et al [2]). For a PML method to be practically useful, it must be stable in time, that is, the solution should not grow exponentially in time. We remark that the well-posedness of the PML system which follows from the theory of symmetric hyperbolic systems allows the exponential growth of the solutions. In $[3,4,2,17]$ the stability of the Cauchy problem of the PML systems is considered separately in each part of the domain where the PML medium property is assumed to be constant.

In this paper we first prove the long-time stability of the initial-boundary value problem of the uniaxial PML system in the PML layer for piecewise constant PML medium properties. The proof is based on an energy argument which is inspired by the method in [3] and the stability analysis of uniaxial PML method in the frequency domain in [11]. We also remark that the stability analysis of the PML method in [20] for the Helmholtz equation is difficult to be used for the time domain analysis as the dependence of the constant in the inf-sup condition on the wave number is not explicit. The second purpose of the paper is to prove the convergence of the time-domain uniaxial PML method. Our technique to prove the PML convergence is different from that for circular PML layer [7]. It is based on the stability estimate for the initialboundary value problem of the PML system in the first part of the paper and an exponential decay estimate of the PML extension in the time domain. This estimate is derived by using the method of Laplace transform, the integral representation of the exterior Dirichlet problem for the Helmholtz equation, and the idea of the complex coordinate stretching.

The layout of the paper is as follows. In section 2 we derive the uniaxial PML formulation for (1.1)-(1.4) by using the method of complex coordinate stretching of Chew and Weedon [12] in the frequency domain, and returning to the time domain by using the inverse Laplace transform. In section 3 we show the stability of the initial boundary value problem of the PML system in the PML layer. In section 4 we prove 
the exponential decay of the PML extension based on the property of the modified Bessel function and the potential theory. The results in Sections 3 and 4 are used to prove the convergence of the PML method in Section 5. In section 6 we present two examples to show the performance of the PML method.

2. The PML equation. For any $s \in \mathbb{C}$ such that $\operatorname{Re}(s)>0$, let $u_{\mathrm{L}}=\mathscr{L}(u)$ and $\mathbf{p}_{\mathrm{L}}=\mathscr{L}(\mathbf{p})$ be the Laplace transform of $u$ and $\mathbf{p}$ in time

$$
u_{\mathrm{L}}(x, s)=\int_{0}^{\infty} e^{-s t} u(x, t) \mathrm{d} t, \quad \mathbf{p}_{\mathrm{L}}(x, s)=\int_{0}^{\infty} e^{-s t} \mathbf{p}(x, t) \mathrm{d} t .
$$

Since $\mathscr{L}\left(\partial_{t} u\right)=s u_{\mathrm{L}}-u_{0}$ and $\mathscr{L}\left(\partial_{t} \mathbf{p}\right)=s \mathbf{p}_{\mathrm{L}}-\mathbf{p}_{0}$, by taking the Laplace transform of (1.1) we get

$$
s u_{\mathrm{L}}-u_{0}=-\operatorname{div}_{\mathrm{L}}+f_{\mathrm{L}}, \quad s \mathbf{p}_{\mathrm{L}}-\mathbf{p}_{0}=-\nabla u_{\mathrm{L}} \quad \text { in } \quad \mathbb{R}^{2} \backslash \bar{D},
$$

where $f_{\mathrm{L}}=\mathscr{L}(f)$.

Let $D$ be contained in the interior of the rectangle $B_{1}=\left\{x \in \mathbb{R}^{2}:\left|x_{1}\right|<\right.$ $\left.L_{1} / 2,\left|x_{2}\right|<L_{2} / 2\right\}$. Let $\Gamma_{1}=\partial B_{1}$ and $\mathbf{n}_{1}$ the unit outer normal to $\Gamma_{1}$. Because $f, u_{0}, \mathbf{p}_{0}$ are all supported inside $B_{1}$, we know that $u_{\mathrm{L}}$ satisfies the Helmholtz equation outside $B_{1}$

$$
-\Delta u_{\mathrm{L}}+s^{2} u_{\mathrm{L}}=0 \quad \text { in } \quad \mathbb{R}^{2} \backslash \bar{B}_{1}
$$

Moreover, (1.3) implies that $u_{\mathrm{L}}$ satisfies the radiation condition

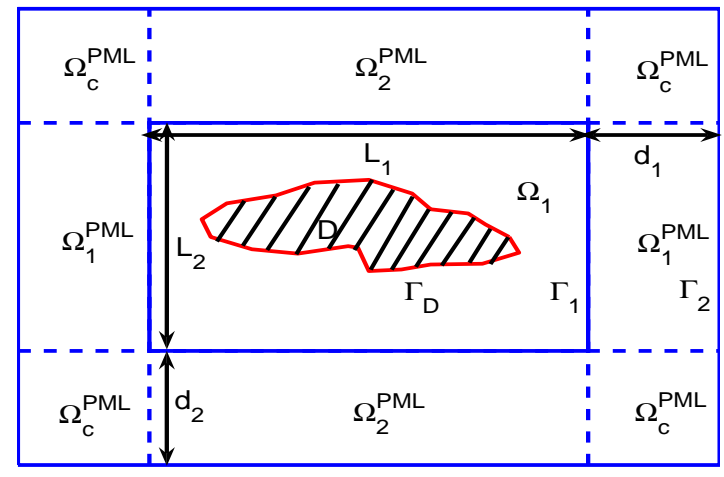

FIG. 2.1. Setting of the scattering problem with the PML layer.

$$
\sqrt{r}\left(\frac{\partial u_{\mathrm{L}}}{\partial r}+s u_{\mathrm{L}}\right) \rightarrow 0, \quad \text { as } r \rightarrow \infty
$$

Let $T_{s}: H^{1 / 2}\left(\Gamma_{1}\right) \rightarrow H^{-1 / 2}\left(\Gamma_{1}\right)$ be the Dirichlet-to-Neumann operator for the Helmholtz equation (2.2) with the complex wave number $s$. For any $\mu \in H^{1 / 2}\left(\Gamma_{1}\right)$, $T_{s}(\mu)=\frac{1}{s} \frac{\partial w}{\partial \mathbf{n}_{1}}$ on $\Gamma_{1}$, where $\mathbf{n}_{1}$ is the unit outer normal to $\Gamma_{1}$ and $w$ is the solution 
of the following exterior Dirichlet problem of the Helmholtz equation

$$
\begin{aligned}
& -\Delta w+s^{2} w=0 \quad \text { in } \mathbb{R}^{2} \backslash \bar{B}_{1}, \\
& w=\mu \quad \text { on } \Gamma_{1}, \\
& \sqrt{r}\left(\frac{\partial w}{\partial r}+s w\right) \rightarrow 0 \quad \text { as } r=|x| \rightarrow \infty .
\end{aligned}
$$

For the complex wave number $s$ with $\operatorname{Re}(s)>0$, it is known by Lax-Milgram lemma that (2.3)-(2.5) has a unique solution $w \in H^{1}\left(\mathbb{R}^{2} \backslash \bar{B}_{1}\right.$ ) (cf. e.g. Nedelec [23, Lemma 2.6.6]). Thus $T_{s}: H^{1 / 2}\left(\Gamma_{1}\right) \rightarrow H^{-1 / 2}\left(\Gamma_{1}\right)$ is well-defined and is a continuous linear operator. We also remark that the well-posedness of (2.3)-(2.5) is also known for the case of real wave number when $\operatorname{Re}(s)=0$ (cf. e.g. McLean [22, Theorem 9.11]).

Since $\mathbf{p}_{0}$ is supported in $B_{1},(2.1)$ implies,

$$
\mathbf{p}_{\mathrm{L}} \cdot \mathbf{n}_{1}+T_{s}\left(u_{\mathrm{L}}\right)=0 \quad \text { on } \Gamma_{1} .
$$

Taking the inverse Laplace transform, we get the Dirichlet-to-Neumann boundary condition

$$
\mathbf{p} \cdot \mathbf{n}_{1}+\mathcal{T}(u)=0 \quad \text { on } \Gamma_{1} \times(0, T),
$$

where $\mathcal{T}=\mathscr{L}^{-1} \circ T_{s} \circ \mathscr{L}$. The acoustic scattering problem (1.1)-(1.4) is reduced to the following problem in the bounded domain $\Omega_{1} \times(0, T)$ with $\Omega_{1}=B_{1} \backslash \bar{D}$,

$$
\begin{aligned}
& \frac{\partial u}{\partial t}=-\operatorname{div} \mathbf{p}+f(x, t), \quad \frac{\partial \mathbf{p}}{\partial t}=-\nabla u \quad \text { in } \Omega_{1} \times(0, T), \\
& \mathbf{p} \cdot \mathbf{n}_{D}=0 \quad \text { on } \quad \Gamma_{D} \times(0, T), \\
& \mathbf{p} \cdot \mathbf{n}_{1}+\mathcal{T}(u)=0 \quad \text { on } \Gamma_{1} \times(0, T), \\
& \left.u\right|_{t=0}=u_{0},\left.\quad \mathbf{p}\right|_{t=0}=\mathbf{p}_{0} .
\end{aligned}
$$

The well-posedness and stability of the reduced problem follows directly from Theorem 2.1 in [7]. In particular, $\mathcal{T}(u) \in L^{2}\left(0, T ; H^{-1 / 2}\left(\Gamma_{1}\right)\right)$.

Now we turn to the introduction of the absorbing PML layer. Let $B_{2}=\left\{x \in \mathbb{R}^{2}\right.$ : $\left.\left|x_{1}\right|<L_{1} / 2+d_{1},\left|x_{2}\right|<L_{2} / 2+d_{2}\right\}$ be the rectangle which contains $B_{1}$. Let

$$
\alpha_{1}\left(x_{1}\right)=1+s^{-1} \sigma_{1}\left(x_{1}\right), \quad \alpha_{2}\left(x_{2}\right)=1+s^{-1} \sigma_{2}\left(x_{2}\right)
$$

be the model medium property, where $\sigma_{j}\left(x_{j}\right)=0$ if $\left|x_{j}\right| \leq L_{j} / 2$ and

$$
\sigma_{j}\left(x_{j}\right)=\sigma_{0} \text { if }\left|x_{j}\right| \geq L_{j} / 2, \quad j=1,2 .
$$

Here $\sigma_{0}>0$ is a constant. Denote by $\tilde{x}_{j}$ the complex coordinate defined by

$$
\tilde{x}_{j}= \begin{cases}x_{j} & \text { if }\left|x_{j}\right|<L_{j} / 2 \\ \int_{0}^{x_{j}} \alpha_{j}(t) \mathrm{d} t & \text { if }\left|x_{j}\right| \geq L_{j} / 2 .\end{cases}
$$

To derive the PML equation, we first notice that by the third Green formula, the solution of the exterior Dirichlet problem (2.3)-(2.5) satisfies

$$
w=-\Psi_{\mathrm{SL}}^{s}(\lambda)+\Psi_{\mathrm{DL}}^{s}(\mu) \quad \text { in } \mathbb{R}^{2} \backslash \bar{B}_{1},
$$


where $\lambda=\left.\frac{\partial w}{\partial \mathbf{n}_{1}}\right|_{\Gamma_{1}}=s T_{s}(\mu) \in H^{-1 / 2}\left(\Gamma_{1}\right)$ is the Neumann trace of $w$ on $\Gamma_{1} . \Psi_{\mathrm{SL}}^{s}, \Psi_{\mathrm{DL}}^{s}$ are respectively the single and double layer potentials [22],

$$
\begin{aligned}
& \Psi_{\mathrm{SL}}^{s}(\lambda)(x)=\int_{\Gamma_{1}} G_{s}(x, y) \lambda(y) \mathrm{d} s(y), \quad \forall \lambda \in H^{-1 / 2}\left(\Gamma_{1}\right), \\
& \Psi_{\mathrm{DL}}^{s}(\mu)(x)=\int_{\Gamma_{1}} \frac{\partial G_{s}(x, y)}{\partial \mathbf{n}_{1}(y)} \mu(y) \mathrm{d} s(y), \quad \forall \mu \in H^{1 / 2}\left(\Gamma_{1}\right) .
\end{aligned}
$$

Here $G_{s}$ is the fundamental solution of the Helmholtz equation satisfying the Sommerfeld radiation condition [28]

$$
G_{s}(x, y)=\frac{1}{2 \pi} K_{0}(s|x-y|),
$$

where $K_{0}(z)$ is the modified Bessel function of order zero.

We follow the method of complex stretching [12] to introduce the PML equation in the frequency domain. For any $z \in \mathbb{C}$, denote by $z^{1 / 2}$ as the analytic branch of $\sqrt{z}$ such that $\operatorname{Re}\left(z^{1 / 2}\right)>0$ for any $z \in \mathbb{C} \backslash(-\infty, 0]$. Let

$$
\rho_{s}(\tilde{x}, y)=\left(s^{2}\left(\left(\tilde{x}_{1}-y_{1}\right)^{2}+\left(\tilde{x}_{2}-y_{2}\right)^{2}\right)\right)^{1 / 2}
$$

be the complex distance and define

$$
\tilde{G}_{s}(x, y)=\frac{1}{2 \pi} K_{0}\left(\rho_{s}(\tilde{x}, y)\right)
$$

It is easy to see that $\tilde{G}_{s}$ is smooth for $x \in \mathbb{R}^{2} \backslash \bar{B}_{1}$ and $y \in \bar{B}_{1}$. Now we can define the modified single and double layer potentials

$$
\begin{aligned}
& \tilde{\Psi}_{\mathrm{SL}}^{s}(\lambda)(x)=\int_{\Gamma_{1}} \tilde{G}_{s}(x, y) \lambda(y) \mathrm{d} s(y), \quad \forall \lambda \in H^{-1 / 2}\left(\Gamma_{1}\right), \\
& \tilde{\Psi}_{\mathrm{DL}}^{s}(\mu)(x)=\int_{\Gamma_{1}} \frac{\partial \tilde{G}_{s}(x, y)}{\partial \mathbf{n}_{1}(y)} \mu(y) \mathrm{d} s(y), \quad \forall \mu \in H^{1 / 2}\left(\Gamma_{1}\right) .
\end{aligned}
$$

It is clear that $\tilde{\Psi}_{\mathrm{SL}}^{s}(\lambda), \tilde{\Psi}_{\mathrm{DL}}^{s}(\mu)$ are smooth in $\mathbb{R}^{2} \backslash \bar{B}_{1}$, and for any $\lambda \in H^{-1 / 2}\left(\Gamma_{1}\right), \mu \in$ $H^{1 / 2}\left(\Gamma_{1}\right)$,

$$
\gamma_{D}^{+} \tilde{\Psi}_{\mathrm{SL}}^{s}(\lambda)=\gamma_{D}^{+} \Psi_{\mathrm{SL}}^{s}(\lambda), \quad \gamma_{D}^{+} \tilde{\Psi}_{\mathrm{DL}}^{s}(\mu)=\gamma_{D}^{+} \Psi_{\mathrm{DL}}^{s}(\mu)
$$

where $\gamma_{D}^{+}: H_{l o c}^{1}\left(\mathbb{R}^{2} \backslash \bar{B}_{1}\right) \rightarrow H^{1 / 2}\left(\Gamma_{1}\right)$ is the trace operator.

For any $\lambda \in H^{-1 / 2}\left(\Gamma_{1}\right), \mu \in H^{1 / 2}\left(\Gamma_{1}\right)$, let $\mathbb{E}(\lambda, \mu)$ be the PML extension given by

$$
\mathbb{E}(\lambda, \mu)=-\tilde{\Psi}_{\mathrm{SL}}^{s}(\lambda)+\tilde{\Psi}_{\mathrm{DL}}^{k}(\mu) \quad \text { for } x \in \mathbb{R}^{2} \backslash \bar{B}_{1} .
$$

By (2.19) and (2.13) we know that

$$
\gamma_{D}^{+} \mathbb{E}(\lambda, \mu)=-\gamma_{D}^{+} \Psi_{\mathrm{SL}}^{k}(\lambda)+\gamma_{D}^{+} \Psi_{\mathrm{DL}}^{k}(\mu)=\gamma_{D}^{+} w=\mu \quad \text { on } \Gamma_{1}
$$

for any $\mu \in H^{1 / 2}\left(\Gamma_{1}\right)$ with $\lambda=s T_{s}(\mu) \in H^{-1 / 2}\left(\Gamma_{1}\right)$. Let

$$
\tilde{u}_{\mathrm{L}}=\mathbb{E}\left(\left.\frac{\partial u_{\mathrm{L}}}{\partial \mathbf{n}_{1}}\right|_{\Gamma_{1}},\left.u_{\mathrm{L}}\right|_{\Gamma_{1}}\right)
$$


be the PML extension of $\left.u_{\mathrm{L}}\right|_{\Gamma_{1}}$ which satisfies $\gamma_{D}^{+} \tilde{u}_{\mathrm{L}}=\left.u_{\mathrm{L}}\right|_{\Gamma_{1}}$ on $\Gamma_{1}$. Since $K_{0}(z)$ decays exponentially on the right half complex plane [8], heuristically $\tilde{u}_{\mathrm{L}}(x)$ will decay exponentially when $x$ is away from $\Gamma_{1}$.

It is obvious that $\tilde{u}_{\mathrm{L}}$ satisfies

$$
-\tilde{\Delta} \tilde{u}_{\mathrm{L}}+s^{2} \tilde{u}_{\mathrm{L}}=0 \quad \text { in } \mathbb{R}^{2} \backslash \bar{B}_{1}
$$

where $\tilde{\Delta}=\widetilde{\operatorname{div}} \tilde{\nabla}$ and $\tilde{\nabla} v=\left(\frac{1}{\alpha_{1}} \frac{\partial v}{\partial x_{1}}, \frac{1}{\alpha_{2}} \frac{\partial v}{\partial x_{2}}\right)^{T}$, $\widetilde{\operatorname{div}} \mathbf{q}=\frac{1}{\alpha_{1}} \frac{\partial \mathbf{q}_{1}}{\partial x_{1}}+\frac{1}{\alpha_{2}} \frac{\partial \mathbf{q}_{2}}{\partial x_{2}}$. Thus we get the PML equation in the frequency domain

$$
-\operatorname{div}\left(A \nabla \tilde{u}_{\mathrm{L}}\right)+s^{2} \alpha_{1} \alpha_{2} \tilde{u}_{\mathrm{L}}=0
$$

where $A=\operatorname{diag}\left(\alpha_{2} / \alpha_{1}, \alpha_{1} / \alpha_{2}\right)$ is a diagonal matrix.

The desired time-domain PML system will be obtained by taking the inverse Laplace transform of (2.21). To that purpose, we introduce new variables

$$
s \tilde{\mathbf{p}}_{\mathrm{L}}^{*}=-\nabla \tilde{u}_{\mathrm{L}}, \quad s \tilde{\mathbf{p}}_{\mathrm{L}}=-A \nabla \tilde{u}_{\mathrm{L}}, \quad s \tilde{u}_{\mathrm{L}}^{*}=\sigma_{1} \sigma_{2} \tilde{u}_{\mathrm{L}}, \quad \forall x \in \mathbb{R}^{2} \backslash \bar{B}_{1} .
$$

The PML equation (2.22) becomes

$$
\operatorname{div} \tilde{\mathbf{p}}_{\mathrm{L}}+s \alpha_{1} \alpha_{2} \tilde{u}_{\mathrm{L}}=0 .
$$

Let, for $x \in \mathbb{R}^{2} \backslash \bar{B}_{1}$,

$$
\tilde{u}=\mathscr{L}^{-1}\left(\tilde{u}_{\mathrm{L}}\right), \tilde{u}^{*}=\mathscr{L}^{-1}\left(\tilde{u}_{\mathrm{L}}^{*}\right), \tilde{\mathbf{p}}=\mathscr{L}^{-1}\left(\tilde{\mathbf{p}}_{\mathrm{L}}\right), \tilde{\mathbf{p}}^{*}=\mathscr{L}^{-1}\left(\tilde{\mathbf{p}}_{\mathrm{L}}^{*}\right),
$$

with $\left.\tilde{u}\right|_{t=0}=0,\left.\tilde{u}^{*}\right|_{t=0}=0,\left.\tilde{\mathbf{p}}\right|_{t=0}=0$, and $\left.\tilde{\mathbf{p}}^{*}\right|_{t=0}=0$. The first two equations in (2.23) imply

$$
s \alpha_{1} \tilde{\mathbf{p}}_{\mathrm{L}, 1}=s \alpha_{2} \tilde{\mathbf{p}}_{\mathrm{L}, 1}^{*}, \quad s \alpha_{2} \tilde{\mathbf{p}}_{\mathrm{L}, 2}=s \alpha_{1} \tilde{\mathbf{p}}_{\mathrm{L}, 2}^{*},
$$

which, since $s \alpha_{1}=s+\sigma_{1}$ and $s \alpha_{2}=s+\sigma_{2}$, by taking the inverse Laplace transform yield

$$
\frac{\partial \tilde{\mathbf{p}}_{1}}{\partial t}+\sigma_{1} \tilde{\mathbf{p}}_{1}=\frac{\partial \tilde{\mathbf{p}}_{1}^{*}}{\partial t}+\sigma_{2} \tilde{\mathbf{p}}_{1}^{*}, \quad \frac{\partial \tilde{\mathbf{p}}_{2}}{\partial t}+\sigma_{2} \tilde{\mathbf{p}}_{2}=\frac{\partial \tilde{\mathbf{p}}_{2}^{*}}{\partial t}+\sigma_{1} \tilde{\mathbf{p}}_{2}^{*} .
$$

Introduce the matrices $\Lambda_{1}(x)=\operatorname{diag}\left(\sigma_{1}\left(x_{1}\right), \sigma_{2}\left(x_{2}\right)\right), \Lambda_{2}(x)=\operatorname{diag}\left(\sigma_{2}\left(x_{2}\right), \sigma_{1}\left(x_{1}\right)\right)$, we can rewrite the above equations in the compact form

$$
\frac{\partial \tilde{\mathbf{p}}}{\partial t}+\Lambda_{1} \tilde{\mathbf{p}}=\frac{\partial \tilde{\mathbf{p}}^{*}}{\partial t}+\Lambda_{2} \tilde{\mathbf{p}}_{2}^{*}
$$

By taking the inverse Laplace transform in the first and third equation in (2.23) we have

$$
\frac{\partial \tilde{\mathbf{p}}^{*}}{\partial t}+\nabla \tilde{u}=0, \quad \frac{\partial \tilde{u}^{*}}{\partial t}=\sigma_{1} \sigma_{2} \tilde{u} .
$$

Finally by taking the inverse Laplace transform in (2.24) we get

$$
\frac{\partial \tilde{u}}{\partial t}+\operatorname{div} \tilde{\mathbf{p}}+\left(\sigma_{1}+\sigma_{2}\right) \tilde{u}+\tilde{u}^{*}=0 .
$$

Since $\tilde{u}=u, \tilde{\mathbf{p}}=\mathbf{p}$ on $\Gamma_{1}, \tilde{u}, \tilde{\mathbf{p}}$ can be viewed as the extension of the solution $(u, \mathbf{p})$ of the problem (1.1)-(1.4). Moreover, since $\sigma_{1}=\sigma_{2}=0$ inside the rectangle $B_{1}$, 
if we set $\tilde{u}=u, \tilde{\mathbf{p}}=\mathbf{p}, \tilde{u}^{*}=0, \tilde{\mathbf{p}}^{*}=\mathbf{p}$ in $\left[B_{1} \backslash \bar{D}\right] \times(0, T)$, then $\left(\tilde{u}, \tilde{\mathbf{p}}, \tilde{u}^{*}, \tilde{\mathbf{p}}^{*}\right)$ satisfies (2.26), (2.27), and, instead of (2.28),

$$
\frac{\partial \tilde{u}}{\partial t}+\operatorname{div} \tilde{\mathbf{p}}+\left(\sigma_{1}+\sigma_{2}\right) \tilde{u}+\tilde{u}^{*}=f .
$$

We summarize the above consideration in the following lemma.

Lemma 2.1. Let $(u, \mathbf{p})$ be the solution of the problem (1.1)-(1.4) which is extended to be $(\tilde{u}, \tilde{\mathbf{p}})$ outside $B_{1}$ according to (2.25). Let $\left(\tilde{u}^{*}, \tilde{\mathbf{p}}^{*}\right)$ be defined in (2.25) for $x \in \mathbb{R}^{2} \backslash \bar{B}_{1}$ and $\left(\tilde{u}^{*}, \tilde{\mathbf{p}}^{*}\right)=(0, \mathbf{p})$ for $x \in B_{1}$. Then $\left(\tilde{u}, \tilde{\mathbf{p}}, \tilde{u}^{*}, \tilde{\mathbf{p}}^{*}\right)$ satisfies the PML system (2.29), (2.26)-(2.27) outside $\bar{D}$ and satisfies the initial and boundary conditions $\left.\tilde{u}\right|_{t=0}=u_{0},\left.\tilde{\mathbf{p}}\right|_{t=0}=\mathbf{p}_{0},\left.\tilde{u}^{*}\right|_{t=0}=0,\left.\tilde{\mathbf{p}}^{*}\right|_{t=0}=\mathbf{p}_{0}$ in $\mathbb{R}^{2} \backslash \bar{D}$ and $\tilde{\mathbf{p}} \cdot \mathbf{n}_{D}=0$ on $\Gamma_{D} \times$ $(0, T)$.

We define the following initial-boundary value problem for $\left(\hat{u}, \hat{\mathbf{p}}, \hat{u}^{*}, \hat{\mathbf{p}}^{*}\right)$ which is referred as the PML problem in the rest of this paper, where $\Omega_{2}=B_{2} \backslash \bar{D}$,

$$
\begin{aligned}
& \frac{\partial \hat{u}}{\partial t}+\operatorname{div} \hat{\mathbf{p}}+\left(\sigma_{1}+\sigma_{2}\right) \hat{u}+\hat{u}^{*}=f, \quad \frac{\partial \hat{u}^{*}}{\partial t}=\sigma_{1} \sigma_{2} \hat{u} \\
& \frac{\partial \hat{\mathbf{p}}^{*}}{\partial t}+\nabla \hat{u}=0, \quad \frac{\partial \hat{\mathbf{p}}}{\partial t}+\Lambda_{1} \hat{\mathbf{p}}=\frac{\partial \hat{\mathbf{p}}^{*}}{\partial t}+\Lambda_{2} \hat{\mathbf{p}}^{*} \quad \text { in } \Omega_{2} \times(0, T), \\
& \hat{\mathbf{p}} \cdot \mathbf{n}_{D}=0 \quad \text { on } \quad \Gamma_{D} \times(0, T), \quad \hat{u}=0 \quad \text { on } \Gamma_{2} \times(0, T), \\
& \left.\hat{u}\right|_{t=0}=u_{0},\left.\quad \hat{\mathbf{p}}\right|_{t=0}=\mathbf{p}_{0},\left.\quad \hat{u}^{*}\right|_{t=0}=0,\left.\quad \hat{\mathbf{p}}^{*}\right|_{t=0}=\mathbf{p}_{0} \quad \text { in } \Omega_{2} .
\end{aligned}
$$

By the construction of the PML problem, $(\hat{u}, \hat{\mathbf{p}})$ is designed to approximate the solution $(u, \mathbf{p})$ of the original scattering problem (1.1)-(1.4) in the domain $\Omega_{1} \times(0, T)$.

Notice that (2.30)-(2.33) is a first order symmetric hyperbolic system whose wellposedness follows from the standard theory (see e.g. Chen [6]). Here we state the well-posedness of the PML problem (2.30)-(2.33) and omit the proof.

Theorem 2.2. Let $f \in L^{\infty}\left(0, T ; L^{2}\left(\Omega_{1}\right)\right)$. Assume that $u_{0} \in H^{1}\left(\Omega_{2}\right), \mathbf{p}_{0} \in$ $H\left(\operatorname{div} ; \Omega_{2}\right)$ are supported in $B_{1}$ and satisfy the compatibility conditions $\mathbf{p}_{0} \cdot \mathbf{n}_{D}=0$, $\nabla u_{0} \cdot \mathbf{n}_{D}=0$ on $\Gamma_{D}$. Then the PML problem (2.30)-(2.33) has a unique strong solution $\left(\hat{u}, \hat{\mathbf{p}}, \hat{u}^{*}, \hat{\mathbf{p}}^{*}\right)$ satisfying

$$
\begin{aligned}
& \hat{u} \in L^{\infty}\left(0, T ; H^{1}\left(\Omega_{2}\right)\right) \cup W^{1, \infty}\left(0, T ; L^{2}\left(\Omega_{2}\right)\right), \quad \hat{u}^{*} \in W^{1, \infty}\left(0, T ; L^{2}\left(\Omega_{2}\right)\right), \\
& \hat{\mathbf{p}} \in L^{\infty}\left(0, T ; H\left(\operatorname{div} ; \Omega_{2}\right)\right) \cup W^{1, \infty}\left(0, T ; L^{2}\left(\Omega_{2}\right)\right), \quad \hat{\mathbf{p}}^{*} \in W^{1, \infty}\left(0, T ; L^{2}\left(\Omega_{2}\right)\right) .
\end{aligned}
$$

3. The stability of the PML system. In this section we consider the stability of the initial-boundary value problem of the following PML system in $\Omega^{\mathrm{PML}} \times(0, T)$, where $\Omega^{\mathrm{PML}}=\Omega_{2} \backslash \bar{\Omega}_{1}$,

$$
\begin{array}{ll}
\frac{\partial \phi}{\partial t}+\operatorname{div} \Phi+\left(\sigma_{1}+\sigma_{2}\right) \phi+\phi^{*}=f_{1}, \quad \frac{\partial \phi^{*}}{\partial t}=\sigma_{1} \sigma_{2} \phi & \text { in } \Omega^{\mathrm{PML}} \times(0, T), \\
\frac{\partial \Phi^{*}}{\partial t}+\nabla \phi=f_{2}, \quad \frac{\partial \Phi}{\partial t}+\Lambda_{1} \Phi=\frac{\partial \Phi^{*}}{\partial t}+\Lambda_{2} \Phi^{*} & \text { in } \Omega^{\mathrm{PML}} \times(0, T), \\
\phi=0 \quad \text { on } \partial \Omega^{\mathrm{PML}} \times(0, T), & \\
\left.\phi\right|_{t=0}=0,\left.\quad \Phi\right|_{t=0}=0,\left.\quad \phi^{*}\right|_{t=0}=0,\left.\quad \Phi^{*}\right|_{t=0}=0 & \text { in } \Omega^{\mathrm{PML}} .
\end{array}
$$

Here we assume that $f_{1}, f_{2} \in H^{1}\left(0, T ; L^{2}\left(\Omega_{2}\right)\right)$. We denote (see Figure 2.1)

$$
\begin{aligned}
& \Omega_{1}^{\mathrm{PML}}=\left\{x \in \Omega^{\mathrm{PML}}:\left|x_{1}\right|>L_{1} / 2, \quad\left|x_{2}\right|<L_{2} / 2\right\}, \\
& \Omega_{2}^{\mathrm{PML}}=\left\{x \in \Omega^{\mathrm{PML}}:\left|x_{1}\right|<L_{1} / 2, \quad\left|x_{2}\right|>L_{2} / 2\right\}, \\
& \Omega_{c}^{\mathrm{PML}}=\left\{x \in \Omega^{\mathrm{PML}}:\left|x_{1}\right|>L_{1} / 2, \quad\left|x_{2}\right|>L_{2} / 2\right\} .
\end{aligned}
$$


We first show the following estimate for the stability of the initial-boundary value problem of PML system.

Theorem 3.1. Let $\left(\phi, \Phi, \phi^{*}, \Phi^{*}\right)$ be the solution of the PML problem (3.1)-(3.4), we have the following stability estimate

$$
\begin{aligned}
& \max _{0 \leq t \leq T}\left(\left\|\partial_{t} \phi+\sigma_{0} \phi\right\|_{L^{2}\left(\Omega^{\mathrm{PML}}\right)}+\left\|\partial_{t} \Phi\right\|_{L^{2}\left(\Omega^{\mathrm{PML}}\right)}+\sum_{j=1}^{2}\left\|\sigma_{0} \Phi_{j}\right\|_{L^{2}\left(\Omega_{j}^{\mathrm{PML}}\right)}\right) \\
\leq & C\left(\left\|f_{1}(\cdot, 0)\right\|_{L^{2}\left(\Omega^{\mathrm{PML}}\right)}+\left\|f_{2}(\cdot, 0)\right\|_{L^{2}\left(\Omega^{\mathrm{PML}}\right)}\right) \\
+ & C \int_{0}^{T}\left(\left\|\partial_{t} f_{1}\right\|_{L^{2}\left(\Omega^{\mathrm{PML}}\right)}+\left\|\partial_{t} f_{2}+\sigma_{0} f_{2}\right\|_{L^{2}\left(\Omega^{\mathrm{PML}}\right)}\right) \mathrm{d} t,
\end{aligned}
$$

where the constant $C$ is independent of $\sigma_{0}$ and $T$.

Proof. We differentiate the first equation in (3.1) in time $t$ and use the second equation in (3.1) to obtain

$$
\frac{\partial^{2} \phi}{\partial t^{2}}+\operatorname{div} \frac{\partial \Phi}{\partial t}+\left(\sigma_{1}+\sigma_{2}\right) \frac{\partial \phi}{\partial t}+\sigma_{1} \sigma_{2} \phi=\partial_{t} f_{1} \quad \text { in } \Omega^{\mathrm{PML}} \times(0, T) .
$$

Multiplying the above equation by $\partial_{t} \phi+\sigma_{0} \phi$ and integrating over $\Omega^{\mathrm{PML}}$, we have

$$
\begin{aligned}
& \frac{1}{2} \frac{\mathrm{d}}{\mathrm{d} t}\left\|\partial_{t} \phi+\sigma_{0} \phi\right\|_{L^{2}\left(\Omega^{\mathrm{PML}}\right)}^{2}+\int_{\Omega^{\mathrm{PML}}} \operatorname{div} \frac{\partial \Phi}{\partial t}\left(\partial_{t} \phi+\sigma_{0} \phi\right) \mathrm{d} x \\
+ & \int_{\Omega^{\mathrm{PML}}}\left[\left(\sigma_{1}+\sigma_{2}-\sigma_{0}\right) \partial_{t} \phi+\sigma_{1} \sigma_{2} \phi\right]\left(\partial_{t} \phi+\sigma_{0} \phi\right) \mathrm{d} x=\int_{\Omega^{\mathrm{PML}}} \partial_{t} f_{1}\left(\partial_{t} \phi+\sigma_{0} \phi\right) \mathrm{d} x .
\end{aligned}
$$

Since $\sigma_{1}+\sigma_{2}-\sigma_{0} \geq 0$ in $\Omega^{\mathrm{PML}}$, the integration in time from 0 to $t$ of the third term on the left-hand of above equation is non-negative. Thus

$$
\begin{aligned}
& \frac{1}{2}\left\|\partial_{t} \phi+\sigma_{0} \phi\right\|_{L^{2}\left(\Omega^{\mathrm{PML}}\right)}^{2}+\int_{0}^{t} \int_{\Omega^{\mathrm{PML}}} \operatorname{div} \frac{\partial \Phi}{\partial t}\left(\partial_{t} \phi+\sigma_{0} \phi\right) \mathrm{d} x \mathrm{~d} t \\
\leq & \frac{1}{2}\left\|\left.\partial_{t} \phi\right|_{t=0}\right\|_{L^{2}\left(\Omega^{\mathrm{PML}}\right)}^{2}+\int_{0}^{t} \int_{\Omega^{\mathrm{PML}}} \partial_{t} f_{1}\left(\partial_{t} \phi+\sigma_{0} \phi\right) \mathrm{d} x \mathrm{~d} t .
\end{aligned}
$$

Here we have used that fact that $\phi(\cdot, 0)=0$. Next we differentiate the first equation in (3.2) in time, multiply the first equation in (3.2) by $\sigma_{0}$, and add the two obtained equations

$$
\frac{\partial^{2} \Phi^{*}}{\partial t^{2}}+\sigma_{0} \frac{\partial \Phi^{*}}{\partial t}+\nabla\left(\partial_{t} \phi+\sigma_{0} \phi\right)=\partial_{t} f_{2}+\sigma_{0} f_{2} \quad \text { in } \Omega^{\mathrm{PML}} \times(0, T) .
$$

On the other hand, by the second equation in (3.2) we have

$$
\begin{aligned}
\frac{\partial^{2} \Phi^{*}}{\partial t^{2}}+\sigma_{0} \frac{\partial \Phi^{*}}{\partial t} & =\frac{\partial^{2} \Phi}{\partial t^{2}}+\Lambda_{1} \frac{\partial \Phi}{\partial t}+\left(\sigma_{0} I-\Lambda_{2}\right) \frac{\partial \Phi^{*}}{\partial t} \\
& =\frac{\partial^{2} \Phi}{\partial t^{2}}+\left(\sigma_{0} I+\Lambda_{1}-\Lambda_{2}\right) \frac{\partial \Phi}{\partial t}+\left(\sigma_{0} I-\Lambda_{2}\right)\left(\Lambda_{1} \Phi-\Lambda_{2} \Phi^{*}\right) .
\end{aligned}
$$

Insert the above identity to (3.6) and multiply the obtained equation by $\partial_{t} \Phi$ and integrate over $\Omega^{\mathrm{PML}}$ we obtain

$$
\begin{aligned}
& \frac{1}{2} \frac{\mathrm{d}}{\mathrm{d} t}\left\|\partial_{t} \Phi\right\|_{L^{2}\left(\Omega^{\mathrm{PML}}\right)}^{2}+\int_{\Omega^{\mathrm{PML}}} \nabla\left(\partial_{t} \phi+\sigma_{0} \phi\right) \partial_{t} \Phi+\int_{\Omega^{\mathrm{PML}}}\left(\sigma_{0} I+\Lambda_{1}-\Lambda_{2}\right)\left|\partial_{t} \Phi\right|^{2} \\
+ & \int_{\Omega^{\mathrm{PML}}}\left(\sigma_{0} I-\Lambda_{2}\right)\left(\Lambda_{1} \Phi-\Lambda_{2} \Phi^{*}\right) \partial_{t} \Phi \mathrm{d} x=\int_{\Omega^{\mathrm{PML}}}\left(\partial_{t} f_{2}+\sigma_{0} f_{2}\right) \partial_{t} \Phi \mathrm{d} x .
\end{aligned}
$$


Since $\sigma_{0} I+\Lambda_{1}-\Lambda_{2} \geq 0$ in $\Omega^{\mathrm{PML}}$, we have

$$
\int_{\Omega^{\mathrm{PML}}}\left(\sigma_{0} I+\Lambda_{1}-\Lambda_{2}\right)\left|\partial_{t} \Phi\right|^{2} \mathrm{~d} x \geq 0 .
$$

Notice that $\sigma_{0} I-\Lambda_{2}=0$ in $\Omega_{c}^{\mathrm{PML}}, \sigma_{0} I-\Lambda_{2}=\operatorname{diag}\left(\sigma_{0}, 0\right)$ in $\Omega_{1}^{\mathrm{PML}}$, and $\sigma_{0} I-\Lambda_{2}=$ $\operatorname{diag}\left(0, \sigma_{0}\right)$ in $\Omega_{2}^{\mathrm{PML}}$, we obtain that

$$
\int_{\Omega^{\mathrm{PML}}}\left(\sigma I-\Lambda_{2}\right)\left(\Lambda_{1} \Phi-\Lambda_{2} \Phi^{*}\right) \partial_{t} \Phi \mathrm{d} x=\frac{\sigma_{0}^{2}}{2} \frac{\mathrm{d}}{\mathrm{d} t}\left(\left\|\Phi_{1}\right\|_{L^{2}\left(\Omega_{1}^{\mathrm{PML}}\right)}^{2}+\left\|\Phi_{2}\right\|_{L^{2}\left(\Omega_{2}^{\mathrm{PML}}\right)}^{2}\right),
$$

Thus it follows from (3.7) that

$$
\begin{aligned}
& \frac{1}{2}\left(\left\|\partial_{t} \Phi\right\|_{L^{2}\left(\Omega^{\mathrm{PML}}\right)}^{2}+\sum_{j=1}^{2}\left\|\sigma_{0} \Phi_{j}\right\|_{L^{2}\left(\Omega_{j}^{\mathrm{PML}}\right)}^{2}\right)+\int_{0}^{t} \int_{\Omega^{\mathrm{PML}}} \nabla\left(\partial_{t} \phi+\sigma_{0} \phi\right) \partial_{t} \Phi \mathrm{d} x \mathrm{~d} t \\
\leq & \frac{1}{2}\left\|\left.\partial_{t} \Phi\right|_{t=0}\right\|_{L^{2}\left(\Omega^{\mathrm{PML}}\right)}^{2}+\int_{0}^{t} \int_{\Omega^{\mathrm{PML}}}\left(\partial_{t} f_{2}+\sigma_{0} f_{2}\right) \partial_{t} \Phi \mathrm{d} x \mathrm{~d} t
\end{aligned}
$$

By adding (3.5) and (3.8) we have

$$
\begin{aligned}
& \frac{1}{2}\left(\left\|\partial_{t} \phi+\sigma_{0} \phi\right\|_{L^{2}\left(\Omega^{\mathrm{PML}}\right)}^{2}+\left\|\partial_{t} \Phi\right\|_{L^{2}\left(\Omega^{\mathrm{PML}}\right)}^{2}+\sum_{j=1}^{2}\left\|\sigma_{0} \Phi_{j}\right\|_{L^{2}\left(\Omega_{j}^{\mathrm{PML}}\right)}^{2}\right) \\
\leq & \frac{1}{2}\left(\left\|\left.\partial_{t} \phi\right|_{t=0}\right\|_{L^{2}\left(\Omega^{\mathrm{PML}}\right)}^{2}+\left\|\left.\partial_{t} \Phi\right|_{t=0}\right\|_{L^{2}\left(\Omega^{\mathrm{PML}}\right)}^{2}\right) \\
+ & \int_{0}^{t} \int_{\Omega^{\mathrm{PML}}} \partial_{t} f_{1}\left(\partial_{t} \phi+\sigma_{0} \phi\right) \mathrm{d} x \mathrm{~d} t+\int_{0}^{t} \int_{\Omega^{\mathrm{PML}}}\left(\partial_{t} f_{2}+\sigma_{0} f_{2}\right) \partial_{t} \Phi \mathrm{d} x \mathrm{~d} t .
\end{aligned}
$$

This completes the proof by using the Cauchy-Schwarz inequality and the following compatibility conditions

$$
\left.\partial_{t} \phi\right|_{t=0}=f_{1}(x, 0),\left.\quad \partial_{t} \Phi\right|_{t=0}=\left.\partial_{t} \Phi^{*}\right|_{t=0}=f_{2}(x, 0),
$$

which follows from (3.1)-(3.2) and the fact that $\phi(\cdot, 0)=0, \Phi(\cdot, 0)=0$. $\square$

Lemma 3.2. Let $\psi=\partial_{t} \phi+\sigma_{0} \phi$. We have

$$
\max _{0 \leq t \leq T}\left\|\sigma_{0} \phi\right\|_{L^{2}\left(\Omega^{\mathrm{PML}}\right)} \leq \max _{0 \leq t \leq T}\|\psi\|_{L^{2}\left(\Omega^{\mathrm{PML}}\right)} .
$$

Proof. We first notice that since $\phi(\cdot, 0)=0$,

$$
\phi(x, t)=\int_{0}^{t} e^{\sigma_{0}(s-t)} \psi(x, s) \mathrm{d} s .
$$

Now

$$
\begin{aligned}
\left|\sigma_{0} \phi\right|^{2}=\sigma_{0}^{2}\left|\int_{0}^{t} e^{\sigma_{0}(s-t)} \psi(x, s) \mathrm{d} s\right|^{2} & \leq \int_{0}^{t} \sigma_{0} e^{\sigma_{0}(s-t)} \mathrm{d} s \cdot \int_{0}^{t} \sigma_{0} e^{\sigma_{0}(s-t)}|\psi|^{2} \mathrm{~d} s \\
& \leq \int_{0}^{t} \sigma_{0} e^{\sigma_{0}(s-t)}|\psi|^{2} \mathrm{~d} s
\end{aligned}
$$


Thus

$$
\int_{\Omega^{\mathrm{PML}}}\left|\sigma_{0} \phi\right|^{2} \mathrm{~d} x \leq \max _{0 \leq t \leq T}\|\psi\|_{L^{2}\left(\Omega^{\mathrm{PML}}\right)}^{2} \int_{0}^{t} \sigma_{0} e^{\sigma_{0}(s-t)} \mathrm{d} s \leq \max _{0 \leq t \leq T}\|\psi\|_{L^{2}\left(\Omega^{\mathrm{PML}}\right)}^{2} .
$$

This completes the proof. $\square$

The following theorem shows the stability of the PML system in the PML layer.

TheOREM 3.3. Let $\left(\phi, \Phi, \phi^{*}, \Phi^{*}\right)$ be the solution of the PML system (3.1)-(3.4), we have the following stability estimate

$$
\begin{aligned}
& \max _{0 \leq t \leq T}\left(\left\|\partial_{t} \phi\right\|_{L^{2}\left(\Omega^{\mathrm{PML}}\right)}+\left\|\partial_{t} \Phi\right\|_{L^{2}\left(\Omega^{\mathrm{PML}}\right)}+\sigma_{0}^{-1}\left\|\partial_{t} \phi^{*}\right\|_{L^{2}\left(\Omega^{\mathrm{PML}}\right)}+\left\|\partial_{t} \Phi^{*}\right\|_{L^{2}\left(\Omega^{\mathrm{PML}}\right)}\right) \\
\leq & C\left(\left\|f_{1}(\cdot, 0)\right\|_{L^{2}\left(\Omega^{\mathrm{PML}}\right)}+\left\|f_{2}(\cdot, 0)\right\|_{L^{2}\left(\Omega^{\mathrm{PML}}\right)}\right) \\
+ & C \int_{0}^{T}\left(\left\|\partial_{t} f_{1}\right\|_{L^{2}\left(\Omega^{\mathrm{PML}}\right)}+\left\|\partial_{t} f_{2}+\sigma_{0} f_{2}\right\|_{L^{2}\left(\Omega^{\mathrm{PML}}\right)}\right) \mathrm{d} t
\end{aligned}
$$

where the constant $C$ is independent of $\sigma_{0}$ and $T$.

Proof. The estimate for $\partial_{t} \phi$ follows from Lemma 3.2 and Theorem 3.1. The estimate for $\partial_{t} \phi^{*}$ follows easily from the second equation in (3.1), Lemma 3.2 and Theorem 3.1. To estimate $\partial_{t} \Phi^{*}$ we first notice that $\Phi^{*}=\Phi$ in $\Omega_{c}^{\mathrm{PML}}$. In $\Omega_{1}^{\mathrm{PML}}$, we have

$$
\frac{\partial \Phi_{1}^{*}}{\partial t}=\frac{\partial \Phi_{1}}{\partial t}+\sigma_{0} \Phi_{1}, \quad \frac{\partial \Phi_{2}^{*}}{\partial t}+\sigma_{0} \Phi_{2}^{*}=\frac{\partial \Phi_{2}}{\partial t} .
$$

Thus the estimate of $\left\|\partial_{t} \Phi_{1}^{*}\right\|_{L^{2}\left(\Omega_{1}^{\mathrm{PML}}\right)}$ can be proved by using Theorem 3.1. The estimate of $\left\|\partial_{t} \Phi_{2}^{*}\right\|_{L^{2}\left(\Omega_{1}^{\mathrm{PML}}\right)}$ follows from Theorem 3.1 and the augument in Lemma 3.2. Similarly we can bound $\left\|\partial_{t} \Phi^{*}\right\|_{L^{2}\left(\Omega_{2}^{\mathrm{PML}}\right)}$. This completes the proof.

We remark that our stability analysis is inspired by the energy analysis in [3] in which the stability is proved separately for the PML system in the unbounded domain parallel to the axises and in the unbounded corner domain. Here we show the stability of the PML system in the whole truncated PML layer which will be useful for the convergence analysis of the time domain PML method in this paper.

4. Exponential decay of the PML extension. In this section we show the exponential decay of the PML extension in the time domain which is the inverse Laplace transform of $\tilde{u}_{\mathrm{L}}$ in $(2.21)$

$$
\tilde{u}=\mathscr{L}^{-1}\left(\mathbb{E}\left(\left.\frac{\partial u_{\mathrm{L}}}{\partial \mathbf{n}_{1}}\right|_{\Gamma_{1}},\left.u_{\mathrm{L}}\right|_{\Gamma_{1}}\right)\right) .
$$

We start with the following elementary lemma.

Lemma 4.1. For any $z_{1}=a_{1}+\mathbf{i} b_{1}, z_{2}=a_{2}+\mathbf{i} b_{2}$ with $a_{1}, b_{1}, a_{2}, b_{2} \in \mathbb{R}$ such that $b_{1}^{2}+b_{2}^{2}>0$, we have

$$
\operatorname{Re}\left[\left(z_{1}^{2}+z_{2}^{2}\right)^{1 / 2}\right] \geq \frac{\left|a_{1} b_{1}+a_{2} b_{2}\right|}{\sqrt{b_{1}^{2}+b_{2}^{2}}}
$$

Proof. For any $a, b \in \mathbb{R}$ we know that

$$
\operatorname{Re}\left[(a+\mathbf{i} b)^{1 / 2}\right]=\sqrt{\frac{a+\sqrt{a^{2}+b^{2}}}{2}} .
$$


Here we used the convention that $z^{1 / 2}$ is the analytic branch of $\sqrt{z}$ such that $\operatorname{Re}\left(z^{1 / 2}\right)>0$ for any $z \in \mathbb{C} \backslash(-\infty, 0]$. It is easy to check that $\operatorname{Re}\left[(a+\mathbf{i} b)^{1 / 2}\right]$ is a increasing function in $a \in \mathbb{R}$. Let $z_{1}^{2}+z_{2}^{2}=a+\mathbf{i} b$, then

$$
a+\mathbf{i} b=\left(\frac{a_{1} b_{1}+a_{2} b_{2}}{\sqrt{b_{1}^{2}+b_{2}^{2}}}+\mathbf{i} \sqrt{b_{1}^{2}+b_{2}^{2}}\right)^{2}+\frac{\left(a_{2} b_{1}-a_{1} b_{2}\right)^{2}}{b_{1}^{2}+b_{2}^{2}} .
$$

Let $a^{\prime}=a-\frac{\left(a_{2} b_{1}-a_{1} b_{2}\right)^{2}}{b_{1}^{2}+b_{2}^{2}}$, we have

$$
\operatorname{Re}\left[\left(a^{\prime}+\mathbf{i} b\right)^{1 / 2}\right]=\frac{\left|a_{1} b_{1}+a_{2} b_{2}\right|}{\sqrt{b_{1}^{2}+b_{2}^{2}}} .
$$

On the other hand, since $a^{\prime} \leq a$, we know that $\operatorname{Re}\left[(a+\mathbf{i} b)^{1 / 2}\right] \geq \operatorname{Re}\left[\left(a^{\prime}+\mathbf{i} b\right)^{1 / 2}\right]$. This completes the proof.

In the following we will always make the following assumption on the thickness of the PML layer which is rather mild in the practical applications.

(H1) $d_{1}=d_{2}=d$ and $L=\max \left(L_{1}, L_{2}\right) \leq C_{0} d$ for some constant $C_{0}>0$.

We remark that in this paper the aspect ratio of the domain $B_{1}$ is held fixed which indicates that $C_{0}$ is bounded. When the aspect ratio grows, then $C_{0}$ will grow which implies our error estimate in Theorem 5.2 may break down. One possible way to overcome the difficulty is to use a PML layer not of the same thickness in $x_{1}$ and $x_{2}$ directions as required in (H1). In particular, if we require $\frac{d_{1}}{L_{1}}=\frac{d_{2}}{L_{2}}$ which is denoted as $\theta$, then the exponential decay of the PML extension can still be achieved as $\theta \rightarrow \infty$.

By (2.11) and (H1) we know that

$$
\bar{\sigma}:=\int_{0}^{L_{1} / 2+d_{1}} \sigma_{1}(t) \mathrm{d} t=\int_{0}^{L_{2} / 2+d_{2}} \sigma_{2}(t) \mathrm{d} t=\sigma_{0} d .
$$

The following lemma will be used in proving the exponential decay of the modified Bessel function.

Lemma 4.2. Let $s=s_{1}+\mathbf{i} s_{2}, s_{1}>0, s_{2} \in \mathbb{R}$. Then for any $x \in \Gamma_{2}, y \in \Gamma_{1}$, the complex distance in (2.17) satisfies

$$
\left|\operatorname{Re}\left[\rho_{s}(\tilde{x}, y) / s\right]\right| \geq d / \sqrt{2}, \quad \operatorname{Re}\left[\rho_{s}(\tilde{x}, y)\right] \geq \gamma \bar{\sigma},
$$

where $\gamma=1 / \sqrt{1+(1+L / d)^{2}}$.

Proof. Let $z_{j}=\tilde{x}_{j}-y_{j}=\left(x_{j}-y_{j}\right)+s^{-1} x_{j} \hat{\sigma}_{j}\left(x_{j}\right)$, where

$$
\hat{\sigma}_{j}=\frac{1}{x_{j}} \int_{0}^{x_{j}} \sigma_{j}(t) \mathrm{d} t .
$$

Then by Lemma 4.1,

$$
\begin{aligned}
& \left|\operatorname{Re}\left[\rho_{s}(\tilde{x}, y) / s\right]\right| \\
= & \operatorname{Re}\left[\left(\left(\tilde{x}_{1}-y_{1}\right)^{2}+\left(\tilde{x}_{2}-y_{2}\right)^{2}\right)^{1 / 2}\right] \\
= & \operatorname{Re}\left[\left(\left(x_{1}-y_{1}+\frac{s_{1} x_{1} \hat{\sigma}_{1}}{s_{1}^{2}+s_{2}^{2}}-\mathbf{i} \frac{s_{2} x_{1} \hat{\sigma}_{1}}{s_{1}^{2}+s_{2}^{2}}\right)^{2}+\left(x_{2}-y_{2}+\frac{s_{1} x_{2} \hat{\sigma}_{2}}{s_{1}^{2}+s_{2}^{2}}-\mathbf{i} \frac{s_{2} x_{2} \hat{\sigma}_{2}}{s_{1}^{2}+s_{2}^{2}}\right)^{2}\right)^{1 / 2}\right] \\
\geq & \frac{\left|\left(x_{1}-y_{1}+\frac{s_{1} x_{1} \hat{\sigma}_{1}}{s_{1}^{2}+s_{2}^{2}}\right) \frac{s_{2} x_{1} \hat{\sigma}_{1}}{s_{1}^{2}+s_{2}^{2}}+\left(x_{2}-y_{2}+\frac{s_{1} x_{2} \hat{\sigma}_{2}}{s_{1}^{2}+s_{2}^{2}}\right) \frac{s_{2} x_{2} \hat{\sigma}_{2}}{s_{1}^{2}+s_{2}^{2}}\right|}{\sqrt{\frac{s_{2}^{2}\left(x_{1} \hat{\sigma}_{1}\right)^{2}}{\left(s_{1}^{2}+s_{2}^{2}\right)^{2}}+\frac{s_{2}^{2}\left(x_{2} \hat{\sigma}_{2}\right)^{2}}{\left(s_{1}^{2}+s_{2}^{2}\right)^{2}}}} .
\end{aligned}
$$


For any $x \in \Gamma_{2}, y \in \Gamma_{1}$, it is easy to see that $x_{j} \hat{\sigma}_{j}\left(x_{j}-y_{j}\right) \geq 0$. Thus for any $x \in \Gamma_{2}, y \in \Gamma_{1}$,

$$
\left|\operatorname{Re}\left[\rho_{s}(\tilde{x}, y) / s\right]\right| \geq \frac{\left|x_{1}-y_{1}\right|\left|x_{1} \hat{\sigma}_{1}\right|+\left|x_{2}-y_{2}\right|\left|x_{2} \hat{\sigma}_{2}\right|}{\sqrt{\left(x_{1} \hat{\sigma}_{1}\right)^{2}+\left(x_{2} \hat{\sigma}_{2}\right)^{2}}} \geq \frac{d \cdot \bar{\sigma}}{\sqrt{2} \bar{\sigma}}=\frac{d}{\sqrt{2}} .
$$

This shows the first estimate. To show the second estimate, let $z_{j}=s\left(\tilde{x}_{j}-y_{j}\right)=$ $s\left(x_{j}-y_{j}\right)+x_{j} \hat{\sigma}_{j}\left(x_{j}\right)$, Again by Lemma 4.1 ,

$$
\begin{aligned}
& \operatorname{Re}\left[\rho_{s}(\tilde{x}, y)\right] \\
= & \operatorname{Re}\left[\left(s^{2}\left(\left(\tilde{x}_{1}-y_{1}\right)^{2}+\left(\tilde{x}_{2}-y_{2}\right)^{2}\right)\right)^{1 / 2}\right] \\
\geq & \frac{\left|\left(s_{1}\left(x_{1}-y_{1}\right)+x_{1} \hat{\sigma}_{1}\right)\left(s_{2}\left(x_{1}-y_{1}\right)\right)+\left(s_{1}\left(x_{2}-y_{2}\right)+x_{2} \hat{\sigma}_{2}\right)\left(s_{2}\left(x_{2}-y_{2}\right)\right)\right|}{\sqrt{s_{2}^{2}\left(x_{1}-y_{1}\right)^{2}+s_{2}^{2}\left(x_{2}-y_{2}\right)^{2}}} .
\end{aligned}
$$

Thus for any $x \in \Gamma_{2}, y \in \Gamma_{1}$,

$$
\operatorname{Re}\left[\rho_{s}(\tilde{x}, y)\right] \geq \frac{\left|x_{1} \hat{\sigma}_{1}\right|\left|x_{1}-y_{1}\right|+\left|x_{2} \hat{\sigma}_{2}\right|\left|x_{2}-y_{2}\right|}{\sqrt{\left(x_{1}-y_{1}\right)^{2}+\left(x_{2}-y_{2}\right)^{2}}} .
$$

If $x_{1}= \pm\left(L_{1} / 2+d\right) \in \Gamma_{2}$, we have $\left|x_{1} \hat{\sigma}_{1}\right|=\bar{\sigma}$ and $\frac{\left|x_{2}-y_{2}\right|}{\left|x_{1}-y_{1}\right|} \leq \frac{L_{2}+d}{d} \leq 1+\frac{L}{d}$. Consequently we deduce

$$
\operatorname{Re}\left[\rho_{s}(\tilde{x}, y)\right] \geq \frac{\bar{\sigma}}{\sqrt{1+(1+L / d)^{2}}}=\gamma \bar{\sigma}
$$

Similarly we can show the same estimate for $x_{2}= \pm\left(L_{2} / 2+d\right) \in \Gamma_{2}$. This completes the proof. $\square$

We need the modified Bessel function $K_{\nu}(z)$ of order $\nu, \nu \in \mathbb{C}$, which is the solution of the differential equation

$$
z^{2} \frac{\mathrm{d}^{2} w}{\mathrm{~d} z^{2}}+z \frac{\mathrm{d} w}{\mathrm{~d} z}-\left(z^{2}+\nu^{2}\right) w=0
$$

satisfying the asymptotic behavior $K_{\nu}(z) \sim \mathbf{i}\left(\frac{\pi}{2 z}\right)^{1 / 2} e^{-z-\frac{\pi}{4} \mathbf{i}}$ as $|z| \rightarrow \infty$. We refer to the treatise Watson [28] for extensive studies on the special function $K_{\nu}(z)$. We recall the Schläfli integral representation formula [28, P. 181], for $z \in \mathbb{C}$ such that $|\arg z|<\pi / 2$,

$$
K_{\nu}(z)=\int_{0}^{\infty} e^{-z \cosh t} \cosh \nu t \mathrm{~d} t .
$$

This implies easily the following lemma.

Lemma 4.3. For any $\nu \in \mathbb{R}, \theta_{2} \geq \theta_{1}>0$, we have

$$
K_{\nu}\left(\theta_{2}\right) \leq e^{-\left(\theta_{2}-\theta_{1}\right)} K_{\nu}\left(\theta_{1}\right) .
$$

The following lemma on the estimates of the fundamental solution $\tilde{G}_{s}$ of the PML equation will play an important role in the analysis in this paper.

LEMma 4.4. Let $\gamma, \sigma_{0}, d$ be so chosen that

$$
\gamma \bar{\sigma} \geq 1
$$


Then there exists a constant $C>0$ independent of $s, \sigma_{0}, d$ but may depend on the constant $C_{0}$ in (H1) such that for any $x \in \Gamma_{2}, y \in \Gamma_{1}$,

(i) $\left|\tilde{G}_{s}(x, y)\right| \leq C e^{-\gamma \bar{\sigma}}$;

(ii) $\frac{\partial \tilde{G}_{s}}{\partial x_{j}}=s P_{1, j}^{s}+P_{0, j}^{s}$ and $\left|P_{l, j}^{s}\right| \leq C \sigma_{0}\left(1+\sigma_{0} s_{1}^{-1}\right) e^{-\gamma \bar{\sigma}}, \quad l=0,1, j=1,2$;

(iii) $\frac{\partial \tilde{G}_{s}}{\partial y_{j}}=s Q_{1, j}^{s}$ and $\left|Q_{1, j}^{s}\right| \leq C\left(1+\sigma_{0} s_{1}^{-1}\right) e^{-\gamma \bar{\sigma}}, \quad j=1,2$;

(iv) $\frac{\partial^{2} \tilde{G}_{s}}{\partial x_{i} \partial y_{j}}=s^{2} R_{2, i j}^{s}+s R_{1, i j}^{s}+R_{0, i j}^{s}$ and $\left|R_{l, i j}^{s}\right| \leq C \sigma_{0}^{2}\left(1+\sigma_{0} s_{1}^{-1}\right)^{2} e^{-\gamma \bar{\sigma}}, \quad l=$ $0,1,2, i, j=1,2$.

Proof. By the Schläfli integral representation formula (4.1), it is easy to see that $\left|K_{0}(z)\right|<K_{0}(\operatorname{Re}(z))$ for any $z \in \mathbb{C}$ such that $\operatorname{Re}(z)>0$. Thus, by (2.18) and Lemma 4.3 , if $\operatorname{Re}\left[\rho_{s}(\tilde{x}, y)\right] \geq 1$,

$$
\left|\tilde{G}_{s}\right| \leq \frac{1}{2 \pi}\left|K_{0}\left(\rho_{s}(\tilde{x}, y)\right)\right| \leq \frac{1}{2 \pi} K_{0}\left(\operatorname{Re}\left[\rho_{s}(\tilde{x}, y)\right]\right) \leq \frac{1}{2 \pi} K_{0}(1) e^{-\left(\operatorname{Re}\left[\rho_{s}(\tilde{x}, y)\right]-1\right)} .
$$

This proves (i) by Lemma 4.2 and (4.2).

To show (ii) we first notice that

$$
\frac{\partial \tilde{G}_{s}}{\partial x_{j}}=\frac{1}{2 \pi} K_{0}^{\prime}\left(\rho_{s}(\tilde{x}, y)\right) \frac{\left(\tilde{x}_{j}-y_{j}\right)\left(s+\sigma_{j}\right)}{\rho_{s}(\tilde{x}, y) / s}=s P_{1, j}^{s}+P_{0, j}^{s} .
$$

From the identity $K_{0}^{\prime}(z)=-K_{1}(z)$ and Lemma $4.2, P_{1, j}^{s}$ has the following estimate

$$
\begin{aligned}
\left|P_{1, j}^{s}\right| & =\frac{1}{2 \pi}\left|K_{0}^{\prime}\left(\rho_{s}(\tilde{x}, y)\right)\right| \frac{\tilde{x}_{j}-y_{j}}{\rho_{s}(\tilde{x}, y) / s}\left|\leq \frac{1}{2 \pi}\right| K_{1}\left(\rho_{s}(\tilde{x}, y)\right) \mid \frac{L_{j}+d+s_{1}^{-1} \bar{\sigma}}{\left|\operatorname{Re}\left[\rho_{s}(\tilde{x}, y) / s\right]\right|} \\
& \leq \frac{1}{2 \pi} K_{1}(1) e^{-(\gamma \bar{\sigma}-1)} \frac{L_{j}+d+s_{1}^{-1} \bar{\sigma}}{(d / \sqrt{2})} \leq C\left(1+\sigma_{0} s_{1}^{-1}\right) e^{-\gamma \bar{\sigma}} .
\end{aligned}
$$

The estimate for $P_{0, j}^{s}$ follows from the similar argument. Similarly, we can prove (iii) by

$$
\frac{\partial \tilde{G}_{s}}{\partial y_{j}}=\frac{1}{2 \pi} K_{0}^{\prime}\left(\rho_{s}(\tilde{x}, y)\right) \frac{\left(\tilde{x}_{j}-y_{j}\right)(-s)}{\rho_{s}(\tilde{x}, y) / s}=s Q_{1, j}^{s} .
$$

To prove (iv) we note that

$$
\begin{aligned}
\frac{\partial^{2} \tilde{G}_{s}}{\partial x_{i} \partial y_{j}}= & -\frac{1}{2 \pi} K_{1}^{\prime}\left(\rho_{s}(\tilde{x}, y)\right) \frac{-\left(\tilde{x}_{i}-y_{i}\right)\left(\tilde{x}_{j}-y_{j}\right)\left(s+\sigma_{i}\right) s}{\rho_{s}^{2}(\tilde{x}, y) / s^{2}} \\
& -\frac{1}{2 \pi} K_{1}\left(\rho_{s}(\tilde{x}, y)\right) \frac{-\left(\rho_{s} / s\right)^{2} \delta_{i j}\left(s+\sigma_{i}\right)+\left(\tilde{x}_{i}-y_{i}\right)\left(\tilde{x}_{j}-y_{j}\right)\left(s+\sigma_{i}\right)}{\rho_{s}^{3}(\tilde{x}, y) / s^{3}} .
\end{aligned}
$$

By using the identity $K_{1}^{\prime}(z)=-\frac{1}{2}\left(K_{0}(z)+K_{2}(z)\right)$, we have

$$
\begin{aligned}
\left|K_{1}^{\prime}\left(\rho_{s}(\tilde{x}, y)\right)\right| & \leq \frac{1}{2}\left(\left|K_{0}\left(\rho_{s}(\tilde{x}, y)\right)\right|+\left|K_{2}\left(\rho_{s}(\tilde{x}, y)\right)\right|\right) \\
& \leq e^{-(\gamma \bar{\sigma}-1)} \frac{1}{2}\left(K_{0}(1)+K_{2}(1)\right)=e^{-(\gamma \bar{\sigma}-1)}\left|K_{1}^{\prime}(1)\right| .
\end{aligned}
$$


On the other hand, by Lemma $4.2,\left|\rho_{s} / s\right|^{-1} \leq C d^{-1} \leq C \sigma_{0}$, where we have used the assumption (4.2) in the last estimate. Therefore,

$$
\frac{\partial^{2} \tilde{G}_{s}}{\partial x_{i} \partial y_{j}}=s^{2} R_{2, i j}^{s}+s R_{1, i j}^{s}+R_{0, i j}^{s}
$$

where $R_{l, i j}$ has the following estimate

$$
\left|R_{l, i j}^{s}\right| \leq C \sigma_{0}^{2}\left(1+\sigma_{0} s_{1}^{-1}\right)^{2} e^{-\gamma \bar{\sigma}}, \quad l=0,1,2, i, j=1,2 .
$$

This completes the proof.

Now we are in the position to estimate the modified single and double layer potentials $\tilde{\Psi}_{\mathrm{SL}}^{k}, \tilde{\Psi}_{\mathrm{DL}}^{k}$. Throughout the paper we shall use the $H^{1 / 2}\left(\Gamma_{j}\right)$ norm, $j=1,2$,

$$
\|v\|_{H^{1 / 2}\left(\Gamma_{j}\right)}=\left(\left|\Gamma_{j}\right|^{-1}\|v\|_{L^{2}\left(\Gamma_{j}\right)}^{2}+|v|_{\frac{1}{2}, \Gamma_{j}}^{2}\right)^{1 / 2},
$$

where

$$
|v|_{\frac{1}{2}, \Gamma_{j}}^{2}=\int_{\Gamma_{j}} \int_{\Gamma_{j}} \frac{\left|v(x)-v\left(x^{\prime}\right)\right|^{2}}{\left|x-x^{\prime}\right|^{2}} \mathrm{~d} s(x) \mathrm{d} s\left(x^{\prime}\right) .
$$

To estimate the PML solution in time domain, we need the following Lemma.

Lemma 4.5. For any $s_{1}>0$ and $f, g \in L^{2}\left(0, T ; L^{2}(\Gamma)\right)$, we have

$$
\left\|\int_{\Gamma} f * g \mathrm{~d} s_{x}\right\|_{L^{2}(0, T)} \leq e^{s_{1} T}\left(\max _{-\infty<s_{2}<+\infty}\left\|(\mathscr{L} f)\left(s_{1}+\mathbf{i} s_{2}\right)\right\|_{L^{2}(\Gamma)}\right)\|g\|_{L^{2}\left(0, T ; L^{2}(\Gamma)\right)} .
$$

Proof. Let $\tilde{f}, \tilde{g}$ be the extension of $f, g$ in $\mathbb{R}$ such that $\tilde{f}=0, \tilde{g}=0$ outside the interval $(0, T)$. Notice that

$$
\mathscr{L} \tilde{f} \cdot \mathscr{L} \tilde{g}=\mathscr{L}(\tilde{f} * \tilde{g})=\mathscr{F}\left(e^{-s_{1} t}(\tilde{f} * \tilde{g})\right), \quad \mathscr{L} \tilde{g}=\mathscr{F}\left(e^{-s_{1} t} \tilde{g}\right),
$$

where $\mathscr{F}$ is the Fourier transform in $t$. By using Parseval equality we obtain

$$
\begin{aligned}
\int_{0}^{T} e^{-2 s_{1} t}\left|\int_{\Gamma} \tilde{f} * \tilde{g} \mathrm{~d} s_{x}\right|^{2} \mathrm{~d} t & =2 \pi \int_{-\infty}^{+\infty}\left|\int_{\Gamma} \mathscr{L} \tilde{f} \cdot \mathscr{L} \tilde{g} \mathrm{~d} s_{x}\right|^{2} \mathrm{~d} s_{2} \\
& \leq 2 \pi \int_{-\infty}^{+\infty}\|\mathscr{L} \tilde{f}\|_{L^{2}(\Gamma)}^{2}\|\mathscr{L} \tilde{g}\|_{L^{2}(\Gamma)}^{2} \mathrm{~d} s_{2} \\
& \leq 2 \pi \max _{-\infty<s_{2}<+\infty}\|\mathscr{L} \tilde{f}\|_{L^{2}(\Gamma)}^{2} \int_{-\infty}^{+\infty}\|\mathscr{L} \tilde{g}\|_{L^{2}(\Gamma)}^{2} \mathrm{~d} s_{2} \\
& =\max _{-\infty<s_{2}<+\infty}\|\mathscr{L} \tilde{f}\|_{L^{2}(\Gamma)}^{2} \int_{-\infty}^{+\infty} \| e^{-s_{1} t \tilde{g} \|_{L^{2}(\Gamma)}^{2} \mathrm{~d} t} \\
& \leq \max _{-\infty<s_{2}<+\infty}\|\mathscr{L} f\|_{L^{2}(\Gamma)}^{2}\|g\|_{L^{2}\left(0, T ; L^{2}(\Gamma)\right)}^{2} .
\end{aligned}
$$

From the definition of convolution, we have

$$
(\tilde{f} * \tilde{g})(t)=\int_{0}^{t} \tilde{f}(t-s) \tilde{g}(s) \mathrm{d} s=\int_{0}^{t} f(t-s) g(s) \mathrm{d} s=(f * g)(t) .
$$

This completes the proof. 
Lemma 4.6. For any $\mu(x, t) \in H^{2}\left(0, T ; L^{2}\left(\Gamma_{1}\right)\right)$ satisfying $\left.\mu\right|_{t=0}=0,\left.\partial_{t} \mu\right|_{t=0}=0$ on $\Gamma_{1}$, let $\mu_{\mathrm{L}}=\mathscr{L} \mu$ and

$$
v(x, t)=\mathscr{L}^{-1}\left(\tilde{\Psi}_{\mathrm{DL}}^{s}\left(\mu_{\mathrm{L}}\right)\right)=\int_{\Gamma_{1}} \mathscr{L}^{-1}\left(\frac{\partial \tilde{G}_{s}(x, y)}{\partial \mathbf{n}_{1}(y)} \mu_{\mathrm{L}}(y)\right) \mathrm{d} s_{y}
$$

be the double layer potential. Then

$$
\|v\|_{L^{2}\left(0, T ; H^{1 / 2}\left(\Gamma_{2}\right)\right)} \leq C \sigma_{0}^{2} d\left(1+\sigma_{0} T\right)^{2} e^{-\gamma \bar{\sigma}}\|\mu\|_{H^{2}\left(0, T ; L^{2}\left(\Gamma_{1}\right)\right)} .
$$

where the constant $C$ is independent of $\sigma_{0}, d$ and $T$.

Proof. From the definition of norm $H^{1 / 2}\left(\Gamma_{2}\right)$, it is easy to see that

$$
\|v\|_{L^{2}\left(0, T ; H^{1 / 2}\left(\Gamma_{2}\right)\right)} \leq C\|v\|_{L^{2}\left(0, T ; L^{\infty}\left(\Gamma_{2}\right)\right)}+C d\left\|\nabla_{x} v\right\|_{L^{2}\left(0, T ; L^{\infty}\left(\Gamma_{2}\right)\right)} .
$$

For any $x \in \Gamma_{2}$, we have

$$
\begin{aligned}
& v(x, t)=\int_{\Gamma_{1}} \mathscr{L}^{-1}\left(\mathbf{Q}_{1}^{s}(x, y) \cdot \mathbf{n}_{1}(y)\right) * \partial_{t} \mu(y) \mathrm{d} s_{y}, \\
& \nabla_{x} v(x, t)=\int_{\Gamma_{1}} \sum_{l=0}^{2} \mathscr{L}^{-1}\left(R_{l}^{s}(x, y) \cdot \mathbf{n}_{1}(y)\right) * \partial_{t}^{l} \mu(y) \mathrm{d} s_{y},
\end{aligned}
$$

where $\mathbf{Q}_{1}^{s}=\left(Q_{1,1}^{s}, Q_{1,2}^{s}\right)^{T}$ and the matrix $R_{l}^{s}=\left(R_{l, i j}^{s}\right)_{i, j=1}^{2}$ with elements $Q_{1, j}^{s}, R_{l, i j}^{s}$ are given in Lemma 4.4.

Then Lemma 4.5 implies that

$$
\begin{aligned}
& \|v(x, t)\|_{L^{2}(0, T)} \leq C e^{s_{1} T} \max _{s_{2} \in \mathbb{R}}\left\|\mathbf{Q}_{1}^{s}(x, \cdot)\right\|_{L^{2}\left(\Gamma_{1}\right)}\|\mu\|_{H^{1}\left(0, T ; L^{2}\left(\Gamma_{1}\right)\right)}, \\
& \left\|\nabla_{x} v(x, t)\right\|_{L^{2}(0, T)} \leq C e^{s_{1} T} \sum_{l=0}^{2} \max _{s_{2} \in \mathbb{R}}\left\|R_{l}^{s}(x, \cdot)\right\|_{L^{2}\left(\Gamma_{1}\right)}\|\mu\|_{H^{2}\left(0, T ; L^{2}\left(\Gamma_{1}\right)\right)} .
\end{aligned}
$$

By Lemma 4.4 (iii),

$$
\left\|\mathbf{Q}_{1}^{s}(x, \cdot)\right\|_{L^{2}\left(\Gamma_{1}\right)} \leq C\left(1+\sigma_{0} s_{1}^{-1}\right) e^{-\gamma \bar{\sigma}} .
$$

By Lemma 4.4 (iv),

$$
\sum_{l=0}^{2}\left\|R_{l}^{s}(x, \cdot)\right\|_{L^{2}\left(\Gamma_{1}\right)} \leq C \sigma_{0}^{2}\left(1+\sigma_{0} s_{1}^{-1}\right)^{2} e^{-\gamma \bar{\sigma}} .
$$

This completes the proof by letting $s_{1}=T^{-1}$. $\mathrm{C}$

Lemma 4.7. For any $\lambda(x, t) \in H^{1}\left(0, T ; L^{2}\left(\Gamma_{1}\right)\right)$ satisfying $\left.\lambda\right|_{t=0}=0$ on $\Gamma_{1}$, let $\lambda_{\mathrm{L}}=\mathscr{L} \lambda$ and

$$
v(x, t)=\mathscr{L}^{-1}\left(\tilde{\Psi}_{\mathrm{SL}}^{s}\left(\lambda_{\mathrm{L}}\right)\right)=\int_{\Gamma_{1}} \mathscr{L}^{-1}\left(\tilde{G}_{s}(x, y) \lambda_{\mathrm{L}}(y)\right) \mathrm{d} s_{y}
$$

be the single layer potential. Then

$$
\|v\|_{L^{2}\left(0, T ; H^{1 / 2}\left(\Gamma_{2}\right)\right)} \leq C \sigma_{0} d\left(1+\sigma_{0} T\right) e^{-\gamma \bar{\sigma}}\|\lambda\|_{H^{1}\left(0, T ; L^{2}\left(\Gamma_{1}\right)\right)},
$$


where the constant $C$ is independent of $\sigma_{0}, d$ and $T$.

Proof. From the definition of norm $H^{1 / 2}\left(\Gamma_{2}\right)$, it is easy to see that

$$
\|v\|_{L^{2}\left(0, T ; H^{1 / 2}\left(\Gamma_{2}\right)\right)} \leq C\|v\|_{L^{2}\left(0, T ; L^{\infty}\left(\Gamma_{2}\right)\right)}+C d\left\|\nabla_{x} v\right\|_{L^{2}\left(0, T ; L^{\infty}\left(\Gamma_{2}\right)\right)} .
$$

For any $x \in \Gamma_{2}$, we have

$$
\begin{aligned}
& v(x, t)=\int_{\Gamma_{1}} \mathscr{L}^{-1}\left(\tilde{G}_{s}(x, y)\right) * \lambda(y) \mathrm{d} s_{y} \\
& \nabla_{x} v(x, t)=\int_{\Gamma_{1}} \sum_{l=0}^{1} \mathscr{L}^{-1}\left(\mathbf{P}_{l}^{s}(x, y)\right) * \partial_{t}^{l} \lambda(y) \mathrm{d} s_{y},
\end{aligned}
$$

where $\mathbf{P}_{l}^{s}=\left(P_{l, 1}^{s}, P_{l, 2}^{s}\right)^{T}, l=0,1$. Then Lemma 4.5 implies that

$$
\begin{aligned}
& \|v(x, t)\|_{L^{2}(0, T)} \leq C e^{s_{1} T} \max _{s_{2} \in \mathbb{R}}\left\|\tilde{G}_{s}(x, \cdot)\right\|_{L^{2}\left(\Gamma_{1}\right)}\|\lambda\|_{L^{2}\left(0, T ; L^{2}\left(\Gamma_{1}\right)\right)}, \\
& \left\|\nabla_{x} v(x, t)\right\|_{L^{2}(0, T)} \leq C e^{s_{1} T} \sum_{l=0}^{1} \max _{s_{2} \in \mathbb{R}}\left\|\mathbf{P}_{l}^{s}(x, \cdot)\right\|_{L^{2}\left(\Gamma_{1}\right)}\|\lambda\|_{H^{1}\left(0, T ; L^{2}\left(\Gamma_{1}\right)\right)} .
\end{aligned}
$$

By Lemma 4.4 (i) and (ii), for any $x \in \Gamma_{2}$,

$$
\begin{aligned}
& \left\|\tilde{G}_{s}(x, \cdot)\right\|_{L^{2}\left(\Gamma_{1}\right)} \leq C\left\|\tilde{G}_{s}(x, \cdot)\right\|_{L^{\infty}\left(\Gamma_{1}\right)} \leq C e^{-\gamma \bar{\sigma}} \\
& \sum_{l=0}^{1}\left\|\mathbf{P}_{l}^{s}(x, \cdot)\right\|_{L^{2}\left(\Gamma_{1}\right)} \leq C \sum_{l=0}^{1}\left\|\mathbf{P}_{l}^{s}(x, \cdot)\right\|_{L^{\infty}\left(\Gamma_{1}\right)} \leq C \sigma_{0}\left(1+\sigma_{0} s_{1}^{-1}\right) e^{-\gamma \bar{\sigma}} .
\end{aligned}
$$

This completes the proof by letting $s_{1}=T^{-1}$. $\mathrm{c}$

The following theorem which is a direct consequence of Lemmas 4.6-4.7 is the main result of this section.

TheOREM 4.8. For any $\lambda \in H^{1}\left(0, T ; L^{2}\left(\Gamma_{1}\right)\right), \mu \in H^{2}\left(0, T ; L^{2}\left(\Gamma_{1}\right)\right)$ satisfying $\left.\lambda\right|_{t=0}=0,\left.\mu\right|_{t=0}=0,\left.\partial_{t} \mu\right|_{t=0}=0$ on $\Gamma_{1}$, let $\lambda_{\mathrm{L}}=\mathscr{L} \lambda, \mu_{\mathrm{L}}=\mathscr{L} \mu$ and $\mathbb{E}\left(\lambda_{\mathrm{L}}, \mu_{\mathrm{L}}\right)$ be the PML extension defined in (2.20). Then there exists a constant $C>0$ independent of $\sigma_{0}, d, T$ but may depend on $C_{0}$ in (H1), such that

$$
\begin{aligned}
\left\|\mathscr{L}^{-1} \mathbb{E}\left(\lambda_{\mathrm{L}}, \mu_{\mathrm{L}}\right)\right\|_{L^{2}\left(0, T ; H^{1 / 2}\left(\Gamma_{2}\right)\right)} & \leq C \sigma_{0} d\left(1+\sigma_{0} T\right) e^{-\gamma \bar{\sigma}}\|\lambda\|_{H^{1}\left(0, T ; L^{2}\left(\Gamma_{1}\right)\right)} \\
& +C \sigma_{0}^{2} d\left(1+\sigma_{0} T\right)^{2} e^{-\gamma \bar{\sigma}}\|\mu\|_{H^{2}\left(0, T ; L^{2}\left(\Gamma_{1}\right)\right)}
\end{aligned}
$$

5. Convergence analysis. In this section, we consider the convergence of the PML method. We start with following lemma.

Lemma 5.1. For any $w \in L^{2}\left(0, T ; H^{1 / 2}\left(\Gamma_{1}\right)\right)$, we have

$$
-\operatorname{Re} \int_{0}^{T} e^{-2 s_{1} t}\langle\mathcal{T}(w), w\rangle_{\Gamma_{1}} \mathrm{~d} t \geq 0
$$

where $\langle\cdot, \cdot\rangle_{\Gamma_{1}}$ is the duality pairing between $H^{-1 / 2}\left(\Gamma_{1}\right)$ and $H^{1 / 2}\left(\Gamma_{1}\right)$ which is the extension of the inner product of $L^{2}\left(\Gamma_{1}\right)$.

Proof. Let $w_{\mathrm{L}}=\mathscr{L}(w)$, from the definition of Dirichlet-to-Neumann operator $\mathcal{T}$, we know that $\mathcal{T}(w)=\mathscr{L}^{-1}\left(T_{s}\left(w_{\mathrm{L}}\right)\right)$, where $w_{\mathrm{L}}$ satisfies (2.3)-(2.5). By applying the 
first Green identity over the bounded domain $\Omega_{R}=B_{R} \backslash \bar{B}_{1}$, where $R>0$ such that $\bar{B}_{1} \subset B_{R}=\left\{x \in \mathbb{R}^{2}:|x|<R\right\}$, we see that

$$
\left(\frac{1}{s} \nabla w_{\mathrm{L}}, \nabla \bar{w}_{\mathrm{L}}\right)+\left(s w_{\mathrm{L}}, \bar{w}_{\mathrm{L}}\right)+\left\langle T_{s}\left(w_{\mathrm{L}}\right), w_{\mathrm{L}}\right\rangle_{\Gamma_{1}}-\left\langle\frac{1}{s} \frac{\partial w_{\mathrm{L}}}{\partial r}, w_{\mathrm{L}}\right\rangle_{\partial B_{R}}=0 .
$$

Take the real part of (5.1) and notice that

$$
\left|\frac{1}{s} \frac{\partial w}{\partial r}+w\right|^{2}=\left|\frac{1}{s} \frac{\partial w}{\partial r}\right|^{2}+|w|^{2}+2 \operatorname{Re}\left(\frac{1}{s} \frac{\partial w}{\partial r} \bar{w}\right),
$$

we have

$$
\begin{aligned}
\operatorname{Re}\left(\frac{1}{s} \nabla w_{\mathrm{L}}, \nabla \bar{w}_{\mathrm{L}}\right)+\operatorname{Re}\left(s w_{\mathrm{L}}, \bar{w}_{\mathrm{L}}\right)+\operatorname{Re}\left\langle T_{s}\left(w_{\mathrm{L}}\right), w_{\mathrm{L}}\right\rangle_{\Gamma_{1}} \\
+\frac{1}{2}\left\|\frac{1}{s} \frac{\partial w_{\mathrm{L}}}{\partial r}\right\|_{L^{2}\left(\partial B_{R}\right)}^{2}+\frac{1}{2}\left\|w_{\mathrm{L}}\right\|_{L^{2}\left(\partial B_{R}\right)}^{2}=\frac{1}{2}\left\|\frac{1}{s} \frac{\partial w_{\mathrm{L}}}{\partial r}+w_{\mathrm{L}}\right\|_{L^{2}\left(\partial B_{R}\right)}^{2} .
\end{aligned}
$$

Since $s_{1}>0$, the first two terms in (5.2) are positive. By the radiation condition (2.5), the right hand side of (5.2) tends to zero when $R \rightarrow \infty$. This yields that $\operatorname{Re}\left\langle T_{s}\left(w_{\mathrm{L}}\right), w_{\mathrm{L}}\right\rangle_{\Gamma_{1}} \leq 0$, then by Parseval equality, we obtain

$$
\begin{aligned}
-\operatorname{Re} \int_{0}^{T} e^{-2 s_{1} t}\langle\mathcal{T}(w), w\rangle_{\Gamma_{1}} \mathrm{~d} t & =-\operatorname{Re} \int_{-\infty}^{\infty} e^{-2 s_{1} t}\left\langle\mathscr{L}^{-1}\left(T_{s}\left(w_{\mathrm{L}}\right)\right), \mathscr{L}^{-1}\left(w_{\mathrm{L}}\right)\right\rangle_{\Gamma_{1}} \mathrm{~d} t \\
& =-2 \pi \operatorname{Re} \int_{-\infty}^{\infty}\left\langle T_{s}\left(w_{\mathrm{L}}\right), w_{\mathrm{L}}\right\rangle_{\Gamma_{1}} \mathrm{~d} s_{2} \geq 0 .
\end{aligned}
$$

This completes the proof. $\square$

For $\hat{u}_{\mathrm{L}}=\mathscr{L}(\hat{u})$ we define its PML extension as in $(2.21)$,

$$
\tilde{\hat{u}}_{\mathrm{L}}=\mathbb{E}\left(\left.\frac{\partial \hat{u}_{\mathrm{L}}}{\partial \mathbf{n}_{1}}\right|_{\Gamma_{1}},\left.\hat{u}_{\mathrm{L}}\right|_{\Gamma_{1}}\right)
$$

and define the corresponding variables $\left(\tilde{\hat{u}}, \tilde{\hat{\mathbf{p}}}, \tilde{\hat{u}}^{*}, \tilde{\hat{\mathbf{p}}}^{*}\right)$ as in (2.25) which satisfies the PML system in $\mathbb{R}^{2} \backslash \bar{D}$. Let $\left(\hat{u}, \hat{\mathbf{p}}, \hat{u}^{*}, \hat{\mathbf{p}}^{*}\right)$ be the solution of the PML system (2.30)$(2.33)$ in $\Omega^{\mathrm{PML}}$. Take $\phi=\hat{u}-\hat{u}, \Phi=\tilde{\hat{\mathbf{p}}}-\hat{\mathbf{p}}, \phi^{*}=\tilde{\hat{u}}^{*}-\hat{u}^{*}, \Phi^{*}=\tilde{\hat{\mathbf{p}}}^{*}-\hat{\mathbf{p}}^{*}$, then $\left(\phi, \Phi, \phi^{*}, \Phi^{*}\right)$ satisfies the following system

$$
\begin{aligned}
& \frac{\partial \phi}{\partial t}+\operatorname{div} \Phi+\left(\sigma_{1}+\sigma_{2}\right) \phi+\phi^{*}=0, \quad \frac{\partial \phi^{*}}{\partial t}=\sigma_{1} \sigma_{2} \phi \quad \text { in } \Omega^{\mathrm{PML}} \times(0, T), \\
& \frac{\partial \Phi^{*}}{\partial t}+\nabla \phi=0, \quad \frac{\partial \Phi}{\partial t}+\Lambda_{1} \Phi=\frac{\partial \Phi^{*}}{\partial t}+\Lambda_{2} \Phi^{*} \quad \text { in } \Omega^{\mathrm{PML}} \times(0, T), \\
& \phi=0 \quad \text { on } \Gamma_{1} \times(0, T), \quad \phi=\left.\tilde{\hat{u}}\right|_{\Gamma_{2}} \quad \text { on } \Gamma_{2} \times(0, T), \\
& \left.\phi\right|_{t=0}=0,\left.\quad \Phi\right|_{t=0}=0,\left.\quad \phi^{*}\right|_{t=0}=0,\left.\quad \Phi^{*}\right|_{t=0}=0 \quad \text { in } \Omega^{\mathrm{PML}} .
\end{aligned}
$$

Let $\zeta$ be a lifting function in $\Omega^{\mathrm{PML}} \times(0, T)$ such that $\zeta \underset{\sim}{=} \underset{\sim}{0}$ on $\Gamma_{1}$ and $\zeta=\left.\tilde{\hat{u}}\right|_{\Gamma_{2}}$ on $\Gamma_{2}$. It is easy to see that we can choose $\zeta(\cdot, 0)=0$. Then $\left(\tilde{\phi}, \tilde{\phi}^{*}, \Phi, \Phi^{*}\right)$, where $\tilde{\phi}=\phi-\zeta$, $\tilde{\phi}^{*}=\phi^{*}-\int_{0}^{t} \sigma_{1} \sigma_{2} \zeta$ satisfies the PML system (3.1)-(3.4) with

$$
f_{1}=-\partial_{t} \zeta-\left(\sigma_{1}+\sigma_{2}\right) \zeta-\int_{0}^{t} \sigma_{1} \sigma_{2} \zeta, \quad f_{2}=-\nabla \zeta \quad \text { in } \quad \Omega^{\mathrm{PML}} \times(0, T),
$$


and zero initial and boundary values. From Theorem 3.3 we have

$$
\begin{aligned}
& \max _{0 \leq t \leq T}\left(\left\|\partial_{t} \phi\right\|_{L^{2}\left(\Omega^{\mathrm{PML}}\right)}+\left\|\partial_{t} \Phi\right\|_{L^{2}\left(\Omega^{\mathrm{PML}}\right)}\right) \\
\leq & C \int_{0}^{T}\left(\left\|\partial_{t} f_{1}\right\|_{L^{2}\left(\Omega^{\mathrm{PML}}\right)}+\left\|\partial_{t} f_{2}+\sigma_{0} f_{2}\right\|_{L^{2}\left(\Omega^{\mathrm{PML}}\right)}\right) \mathrm{d} t .
\end{aligned}
$$

By (5.3) we have

$$
\|\operatorname{div} \Phi\|_{L^{2}\left(\Omega^{\mathrm{PML}}\right)} \leq\left\|\partial_{t} \phi\right\|_{L^{2}\left(\Omega^{\mathrm{PML}}\right)}+2\left\|\sigma_{0} \phi\right\|_{L^{2}\left(\Omega^{\mathrm{PML}}\right)}+\left\|\phi^{*}\right\|_{L^{2}\left(\Omega^{\mathrm{PML}}\right)} .
$$

Thus by Lemma 3.2 we have

$$
\begin{aligned}
& \max _{0 \leq t \leq T}\left(\|\operatorname{div} \Phi\|_{L^{2}\left(\Omega^{\mathrm{PML}}\right)}+\left\|\partial_{t} \Phi\right\|_{L^{2}\left(\Omega^{\mathrm{PML}}\right)}\right) \\
\leq & C\left(1+\sigma_{0} T\right) \int_{0}^{T}\left(\left\|\partial_{t} f_{1}\right\|_{L^{2}\left(\Omega^{\mathrm{PML}}\right)}+\left\|\partial_{t} f_{2}+\sigma f_{2}\right\|_{L^{2}\left(\Omega^{\mathrm{PML}}\right)}\right) \mathrm{d} t \\
\leq & C\left(1+\sigma_{0} T\right) \sigma_{0}^{2} T^{1 / 2}\left(\|\zeta\|_{H^{2}\left(0, T ; L^{2}\left(\Omega^{\mathrm{PML}}\right)\right)}+\|\zeta\|_{H^{1}\left(0, T ; H^{1}\left(\Omega^{\mathrm{PML}}\right)\right)}\right) .
\end{aligned}
$$

The above estimate is valid for any $\zeta$ implies that

$$
\begin{aligned}
& \max _{0 \leq t \leq T}\left(\|\operatorname{div} \Phi\|_{L^{2}\left(\Omega^{\mathrm{PML}}\right)}+\left\|\partial_{t} \Phi\right\|_{L^{2}\left(\Omega^{\mathrm{PML}}\right)}\right) \\
& \leq C\left(1+\sigma_{0} T\right) \sigma_{0}^{2} T^{1 / 2}\left(\|\tilde{\hat{u}}\|_{H^{2}\left(0, T ; H^{1 / 2}\left(\Gamma_{2}\right)\right)}\right) .
\end{aligned}
$$

Now we are in the position to show the convergence of the time domain PML method in the following theorem.

THEOREM 5.2. Let $(u, \mathbf{p})$ be the solution of the problem (1.1)-(1.4) and $(\hat{u}, \hat{\mathbf{p}}$, $\left.\hat{u}^{*}, \hat{\mathbf{p}}^{*}\right)$ be the solution of the PML problem (2.30)-(2.33). Then there exists a constant $C>0$ independently of $\sigma_{0}, d, T$ but may depend on $C_{0}$ in (H1) such that

$$
\begin{aligned}
& \max _{0 \leq t \leq T}\left(\|u-\hat{u}\|_{L^{2}\left(\Omega_{1}\right)}+\|\mathbf{p}-\hat{\mathbf{p}}\|_{L^{2}\left(\Omega_{1}\right)}\right) \\
\leq & C T^{3 / 2} \sigma_{0}^{3} d\left(1+\sigma_{0} T\right)^{3} e^{-\gamma \bar{\sigma}}\left(\left\|\partial \hat{u} / \partial \mathbf{n}_{1}\right\|_{H^{3}\left(0, T ; L^{2}\left(\Gamma_{1}\right)\right)}+\sigma_{0}\|\hat{u}\|_{H^{4}\left(0, T ; L^{2}\left(\Gamma_{1}\right)\right)}\right) .
\end{aligned}
$$

Proof. From (2.7) and (2.30)-(2.31), we have

$$
\begin{aligned}
& \frac{\partial(u-\hat{u})}{\partial t}+\operatorname{div}(\mathbf{p}-\hat{\mathbf{p}})=0, \quad \text { in } \Omega_{1} \times(0, T), \\
& \frac{\partial(\mathbf{p}-\hat{\mathbf{p}})}{\partial t}+\nabla(u-\hat{u})=0, \quad \text { in } \Omega_{1} \times(0, T) .
\end{aligned}
$$

By testing (5.8) with $v \in H^{1}\left(\Omega_{1}\right)$ and using (2.6) we get

$$
\left(\frac{\partial(u-\hat{u})}{\partial t}, v\right)-(\mathbf{p}-\hat{\mathbf{p}}, \nabla v)-\langle\mathcal{T}(u-\hat{u}), v\rangle_{\Gamma_{1}}=\left\langle\hat{\mathbf{p}} \cdot \mathbf{n}_{1}+\mathcal{T}(\hat{u}), v\right\rangle_{\Gamma_{1}}
$$

Let $w=u-\hat{u}$ and $w^{*}=\int_{0}^{t}(u-\hat{u}) \mathrm{d} t$. From (5.9) we have $\mathbf{p}-\hat{\mathbf{p}}=-\nabla w^{*}$. Thus by taking $v=w$ we have

$$
\frac{1}{2} \frac{\mathrm{d}}{\mathrm{d} t}\left(\|w\|_{L^{2}\left(\Omega_{1}\right)}^{2}+\left\|\nabla w^{*}\right\|_{L^{2}\left(\Omega_{1}\right)}^{2}\right)-\langle\mathcal{T}(w), w\rangle_{\Gamma_{1}}=\left\langle\hat{\mathbf{p}} \cdot \mathbf{n}_{1}+\mathcal{T}(\hat{u}), w\right\rangle_{\Gamma_{1}} .
$$




\section{Denote}

$$
X\left(0, T ; \Omega_{1}\right)=\left\{v \in L^{\infty}\left(0, T ; L^{2}\left(\Omega_{1}\right)\right), v^{*}=\int_{0}^{t} v \mathrm{~d} t \in L^{\infty}\left(0, T ; H^{1}\left(\Omega_{1}\right)\right)\right\},
$$

which is a Banach space with the norm

$$
\|v\|_{X\left(0, T ; \Omega_{1}\right)}=\sup _{0 \leq t \leq T}\left(\|v\|_{L^{2}\left(\Omega_{1}\right)}^{2}+\left\|\nabla v^{*}\right\|_{L^{2}\left(\Omega_{1}\right)}^{2}\right)^{1 / 2}
$$

Define

$$
Y\left(0, T ; \Gamma_{1}\right)=\left\{\varphi: \int_{0}^{T}\langle\varphi, v\rangle_{\Gamma_{1}} \mathrm{~d} t<\infty, \forall v \in X\left(0, T ; \Omega_{1}\right)\right\} .
$$

It is easy to see that $Y\left(0, T ; \Gamma_{1}\right)$ is also a Banach space with the norm

$$
\|\varphi\|_{Y\left(0, T ; \Gamma_{1}\right)}=\sup _{v \in X\left(0, T ; \Omega_{1}\right)} \frac{\left|\int_{0}^{T}\langle\varphi, v\rangle_{\Gamma_{1}} \mathrm{~d} t\right|}{\|v\|_{X\left(0, T ; \Omega_{1}\right)}} .
$$

From (5.11) and Lemma 5.1 we obtain

$$
\left\|e^{-s_{1} t} w\right\|_{X\left(0, T ; \Omega_{1}\right)}^{2} \leq C\left\|e^{-s_{1} t}\left(\hat{\mathbf{p}} \cdot \mathbf{n}_{1}+\mathcal{T}(\hat{u})\right)\right\|_{Y\left(0, T ; \Gamma_{1}\right)}\left\|e^{-s_{1} t} w\right\|_{X\left(0, T ; \Omega_{1}\right)} .
$$

By letting $s_{1} \rightarrow 0$, we get

$$
\sup _{0 \leq t \leq T}\left(\|w\|_{L^{2}\left(\Omega_{1}\right)}+\left\|\nabla w^{*}\right\|_{L^{2}\left(\Omega_{1}\right)}\right) \leq C\left\|\hat{\mathbf{p}} \cdot \mathbf{n}_{1}+\mathcal{T}(\hat{u})\right\|_{Y\left(0, T ; \Gamma_{1}\right)} .
$$

It is clear that $\mathcal{T}(\hat{u})=-\tilde{\hat{\mathbf{p}}} \cdot \mathbf{n}_{1}$ on $\Gamma_{1} \times(0, T)$. Therefore we need to estimate $\|(\hat{\mathbf{p}}-$ $\tilde{\hat{\mathbf{p}}}) \cdot \mathbf{n}_{1}\left\|_{Y\left(0, T ; \Gamma_{1}\right)}=\right\| \Phi \cdot \mathbf{n}_{1} \|_{Y\left(0, T ; \Gamma_{1}\right)}$. Notice that any function $v \in X\left(0, T ; \Omega_{1}\right)$ can be extended to $\Omega^{\mathrm{PML}} \times(0, T)$, such that $v=0$ on $\Gamma_{2} \times(0, T)$ and $\|v\|_{X\left(0, T ; \Omega^{\mathrm{PML}}\right)} \leq$ $C\|v\|_{X\left(0, T ; \Omega_{1}\right)}$. Thus

$$
\left\|\Phi \cdot \mathbf{n}_{1}\right\|_{Y\left(0, T ; \Gamma_{1}\right)} \leq C \sup _{v \in X\left(0, T ; \Omega^{\mathrm{PML}}\right)} \frac{\left|\int_{0}^{T}\left\langle\Phi \cdot \mathbf{n}_{1}, v\right\rangle_{\Gamma_{1}} \mathrm{~d} t\right|}{\|v\|_{X\left(0, T ; \Omega^{\mathrm{PML}}\right)}} .
$$

On the other hand, since $v=0$ on $\Gamma_{2}$, we have

$$
\int_{0}^{T}\left\langle\Phi \cdot \mathbf{n}_{1}, v\right\rangle_{\Gamma_{1}} \mathrm{~d} t=\int_{0}^{T}\left[(\operatorname{div} \Phi, v)_{\Omega^{\mathrm{PML}}}+(\Phi, \nabla v)_{\Omega^{\mathrm{PML}}}\right] \mathrm{d} t .
$$

Integrating by parts we obtain

$$
\begin{aligned}
\int_{0}^{T}(\Phi, \nabla v)_{\Omega^{\mathrm{PML}}} \mathrm{d} t & =\left(\Phi(\cdot, T), \nabla v^{*}(\cdot, T)\right)_{\Omega^{\mathrm{PML}}}-\int_{0}^{T}\left(\partial_{t} \Phi, \nabla v^{*}\right)_{\Omega^{\mathrm{PML}}} \mathrm{d} t \\
& \leq \max _{0 \leq t \leq T}\left\|\nabla v^{*}\right\|_{L^{2}\left(\Omega^{\mathrm{PML}}\right)} \int_{0}^{T}\left\|\partial_{t} \Phi\right\|_{L^{2}\left(\Omega^{\mathrm{PML}}\right)} \mathrm{d} t,
\end{aligned}
$$

where we have used the fact that $\left.\Phi\right|_{t=0}=0$. Therefore,

$$
\left\|\Phi \cdot \mathbf{n}_{1}\right\|_{Y\left(0, T ; \Gamma_{1}\right)} \leq C \int_{0}^{T}\left(\|\operatorname{div} \Phi\|_{L^{2}\left(\Omega^{\mathrm{PML}}\right)}+\left\|\partial_{t} \Phi\right\|_{L^{2}\left(\Omega^{\mathrm{PML}}\right)}\right) \mathrm{d} t
$$


and consequently, by (5.12) and (5.7),

$$
\begin{aligned}
\sup _{0 \leq t \leq T}\left(\|w\|_{L^{2}\left(\Omega_{1}\right)}+\left\|\nabla w^{*}\right\|_{L^{2}\left(\Omega_{1}\right)}\right) & \leq C \int_{0}^{T}\left(\|\operatorname{div} \Phi\|_{L^{2}\left(\Omega^{\mathrm{PML}}\right)}+\left\|\partial_{t} \Phi\right\|_{L^{2}\left(\Omega^{\mathrm{PML}}\right)}\right) \mathrm{d} t \\
& \leq C\left(1+\sigma_{0} T\right) \sigma_{0}^{2} T^{3 / 2}\left(\|\tilde{\hat{u}}\|_{H^{2}\left(0, T ; H^{1 / 2}\left(\Gamma_{2}\right)\right)}\right) .
\end{aligned}
$$

This completes the proof by using Theorem 4.8.

The theorem indicates that for the fixed PML absorbing coefficient $\sigma_{0}$, any error of the time domain PML method can be achieved by enlarging the thickness of the PML layer as $\ln T$ for large $T>0$.

6. Numerical Implementation. In this section, we present three numerical examples to illustrate the performance of the PML method. The computations are carried out in Matlab programming environment. We first briefly describe the numerical method used in our computations.

Denote by $H_{\Gamma_{2}}^{1}\left(\Omega_{2}\right)=\left\{v \in H^{1}\left(\Omega_{2}\right): v=0\right.$ on $\left.\Gamma_{2}\right\}$. The equivalent weak formulation of (2.30)-(2.33) is: Find $u \in L^{2}\left(0, T ; H_{\Gamma_{2}}^{1}\left(\Omega_{2}\right)\right), \mathbf{p} \in L^{2}\left(0, T ; L^{2}\left(\Omega_{2}\right)\right)$, $u^{*} \in L^{2}\left(0, T ; L^{2}\left(\Omega_{2}\right)\right), \mathbf{p}^{*} \in L^{2}\left(0, T ; L^{2}\left(\Omega_{2}\right)\right)$ such that

$$
\begin{aligned}
& \left(\frac{\partial u}{\partial t}, v\right)-(\mathbf{p}, \nabla v)+\left(\left(\sigma_{1}+\sigma_{2}\right) u, v\right)+\left(u^{*}, v\right)=(f, v), \quad \forall v \in H_{\Gamma_{2}}^{1}\left(\Omega_{2}\right), \\
& \left(\frac{\partial \mathbf{p}^{*}}{\partial t}, \mathbf{q}^{*}\right)+\left(\nabla u, \mathbf{q}^{*}\right)=0, \quad \forall \mathbf{q}^{*} \in L^{2}\left(\Omega_{2}\right), \\
& \left(\frac{\partial u^{*}}{\partial t}, v^{*}\right)-\left(\sigma_{1} \sigma_{2} u, v^{*}\right)=0, \quad \forall v^{*} \in H^{1}\left(\Omega_{2}\right), \\
& \left(\frac{\partial \mathbf{p}}{\partial t}, \mathbf{q}\right)+\left(\Lambda_{1} \mathbf{p}, \mathbf{q}\right)=\left(\frac{\partial \mathbf{p}^{*}}{\partial t}, \mathbf{q}\right)+\left(\Lambda_{2} \mathbf{p}^{*}, \mathbf{q}\right), \quad \forall \mathbf{q} \in L^{2}\left(\Omega_{2}\right), \\
& \left.u\right|_{t=0}=u_{0},\left.\quad \mathbf{p}\right|_{t=0}=\mathbf{p}_{0},\left.\quad u^{*}\right|_{t=0}=0,\left.\quad \mathbf{p}^{*}\right|_{t=0}=\mathbf{p}_{0} \quad \text { in } \Omega_{2} .
\end{aligned}
$$

Let $\left\{t_{0}, \cdots, t_{N}\right\}$ be a partition of the time interval $[0, T]$ and $\tau_{n}=t_{n}-t_{n-1}$ be the $n$-th timestep size. Let $\mathcal{M}_{h}$ be a regular triangulation of $\Omega_{2}$. We assume the elements $K \in \mathcal{M}_{h}$ may have one curved edge align with $\Gamma_{D}$ so that $\Omega_{2}=\cup_{K \in \mathcal{M}_{h}} K$. Let $V_{h} \subset H^{1}\left(\Omega_{2}\right)$ be the conforming linear finite element space over $\mathcal{M}_{h}$, and $\stackrel{\circ}{V}_{h}=$ $\left\{v_{h} \in V_{h}: v_{h}=0\right.$ on $\left.\Gamma_{2}\right\}$. Let $W_{h} \subset L^{2}\left(\Omega_{2}\right)$ be the piecewise constant element space over $\mathcal{M}_{h}$.

We use the Crank-Nicolson scheme in time. Denote by

$$
\partial v^{n}=\frac{v^{n}-v^{n-1}}{\tau_{n}}, \quad \bar{v}^{n}=\frac{v^{n}+v^{n-1}}{2},
$$

then the fully discrete scheme of (6.1)-(6.5) reads as follows: Given $\left(U^{n-1}, \mathbf{P}^{n-1}\right.$, $\left.U^{*, n-1}, \mathbf{P}^{*, n-1}\right) \in \stackrel{\circ}{V}_{h} \times\left(W_{h}\right)^{2} \times V_{h} \times\left(W_{h}\right)^{2}$, find $\left(U^{n}, \mathbf{P}^{n}, U^{*, n}, \mathbf{P}^{*, n}\right) \in \stackrel{\circ}{V}_{h} \times\left(W_{h}\right)^{2} \times$ $V_{h} \times\left(W_{h}\right)^{2}$ such that

$$
\begin{aligned}
& \left(\partial U^{n}, V\right)-\left(\overline{\mathbf{P}}^{n}, \nabla V\right)+\left(\left(\sigma_{1}+\sigma_{2}\right) \bar{U}^{n}, V\right)+\left(\bar{U}^{*, n}, V\right)=\left(\hat{f}_{n}, V\right), \quad \forall V \in \stackrel{\circ}{V}_{h}, \\
& \left(\partial \mathbf{P}^{*, n}, \mathbf{Q}^{*}\right)+\left(\nabla \bar{U}^{n}, \mathbf{Q}^{*}\right)=0, \quad \forall \mathbf{Q}^{*} \in\left(W_{h}\right)^{2}, \\
& \left(\partial U^{*, n}, V^{*}\right)-\left(\sigma_{1} \sigma_{2} \bar{U}^{n}, V^{*}\right)=0, \quad \forall V^{*} \in V_{h}, \\
& \left(\partial \mathbf{P}^{n}, \mathbf{Q}\right)+\left(\Lambda_{1} \overline{\mathbf{P}}^{n}, \mathbf{Q}\right)=\left(\partial \mathbf{P}^{*, n}, \mathbf{Q}\right)+\left(\Lambda_{2} \overline{\mathbf{P}}^{*, n}, \mathbf{Q}\right), \quad \forall \mathbf{Q} \in\left(W_{h}\right)^{2},
\end{aligned}
$$


where $\hat{f}_{n}:=\frac{1}{\tau_{n}} \int_{t_{n-1}}^{t_{n}} f(t) \mathrm{d} t$ is the mean value of $f$ over $\left[t_{n-1}, t_{n}\right]$.

Example 1. Let $B_{1}=[-0.5,0.5]^{2}$, the source is located at origin with the source term

$$
f(x, t)=\delta(x) t
$$

which exists for all time. The initial values $u_{0}$ and $\mathbf{p}_{0}$ are taken as zeros. By using the time-domain Green's function, we can get the exact solution excited by this source

$$
u(x, t)=\int_{\Omega} \int_{0}^{t} G_{t-s}(x, y) \partial_{t} f(y, s) \mathrm{d} s \mathrm{~d} y .
$$

The delta function is approximated by the Gaussian function and numerical integration is also used to compute the above convolution.

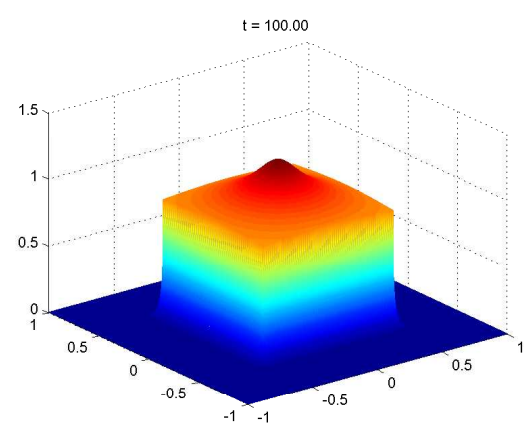

FIG. 6.1. Example 1: numerical solution at final step $T=100.0$ with a point source at origin.

First, we take $L_{1}=L_{2}=1.0$, fix $d_{1}=d_{2}=0.5$ and choose $\sigma_{0}=25$. In the computation, a mesh of 227533 nodes is used which is refined around the boundaries where the coefficients of the PML system are discontinuous and the final time is set to be $T=100.0$. In Figure 6.1, we show the numerical solution at the final step. It is observed that the waves are attenuated in the PML layer without spurious reflection.
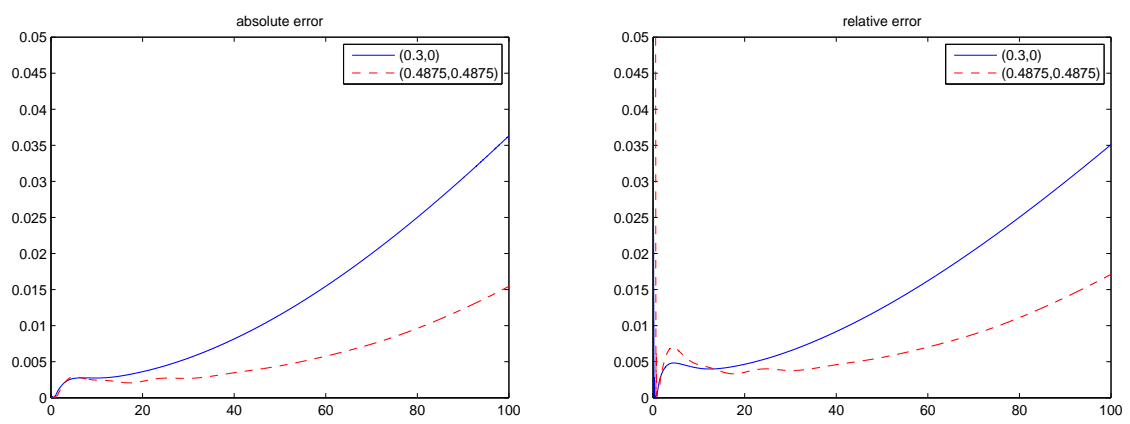

FIG. 6.2. Example 1: The absolute and relative errors of the numerical solutions at points $(0.3,0)$ and $(0.4875,0.4875)$ for different times $\left(d_{1}=d_{2}=0.5\right)$.

In Figure 6.2, we show the absolute and relative errors of the numerical solution at points $(0.3,0)$ and $(0.4875,0.4875)$ for different times. It is observed that the long time stability and convergence hold even for the point very close to the PML interface. 

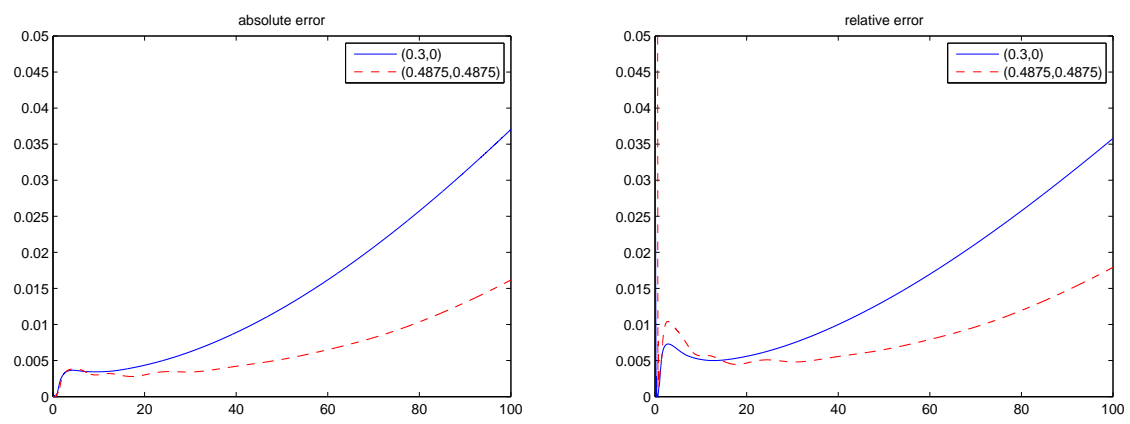

FIG. 6.3. Example 1: The absolute and relative errors of the numerical solutions at points $(0.3,0)$ and $(0.4875,0.4875)$ for different times $\left(d_{1}=d_{2}=0.25\right)$.

Then, we fix $d_{1}=d_{2}=0.25$, choose $\sigma_{0}=50$ and do the numerical experiment again. The mesh of 298113 nodes is used and the final time is also set to be $T=100.0$. Figure 6.3 shows the computational errors at the above points. The long time stability and convergence are also observed.

Example 2. Let $B_{1}=[-0.5,0.5]^{2}$, the source is located at origin with the source term

$$
f(x, t)=\delta(x) \sin (\omega t),
$$

which oscillates with $\omega=1$ for all time. The initial values $u_{0}$ and $\mathbf{p}_{0}$ are taken as zeros. The exact solution can also be computed by using (6.7).
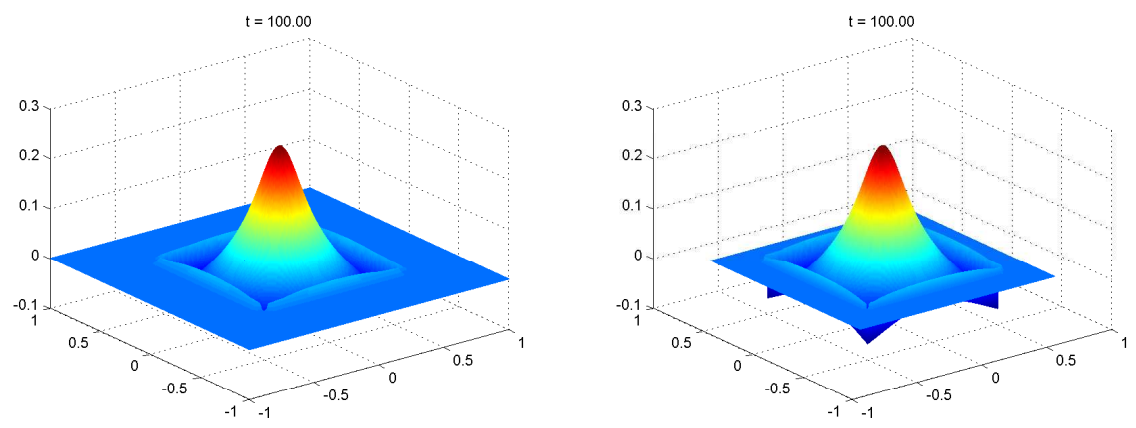

FIG. 6.4. Example 2: The numerical solution at final step $T=100.0$ with an oscillating source at origin (left: $d_{1}=d_{2}=0.5$, right: $d_{1}=d_{2}=0.25$ ).

In this example, we take $L_{1}=L_{2}=1.0$ and choose two sets of PML parameters the same as those in Example 1. We also use the same meshes and set the final time $T=100.0$. Figure 6.4 displays the numerical solutions at the final step. It shows that the waves are absorbed rapidly as they propagate through the PML layer in each case.

In Figure 6.5, we compare the exact solution to the numerical solution at point $(0.4875,0.4875)$ for different times. The solid curve represents the exact solution and the dashed curve the numerical one. It shows a very good agreement between the numerical solutions and the exact ones, even for a long time computation. 

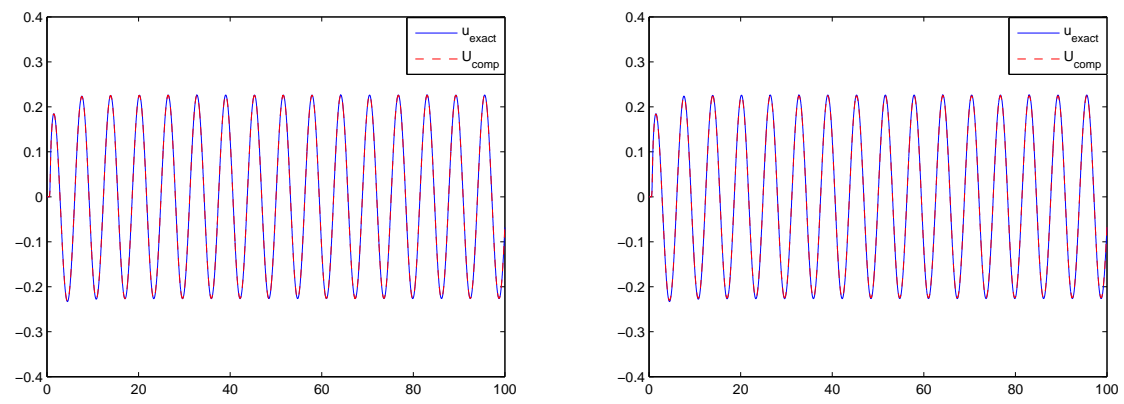

Fig. 6.5. Example 2: The exact and numerical solution at point $(0.4875,0.4875)$ for different times (left: $d_{1}=d_{2}=0.5$, right: $d_{1}=d_{2}=0.25$ ).
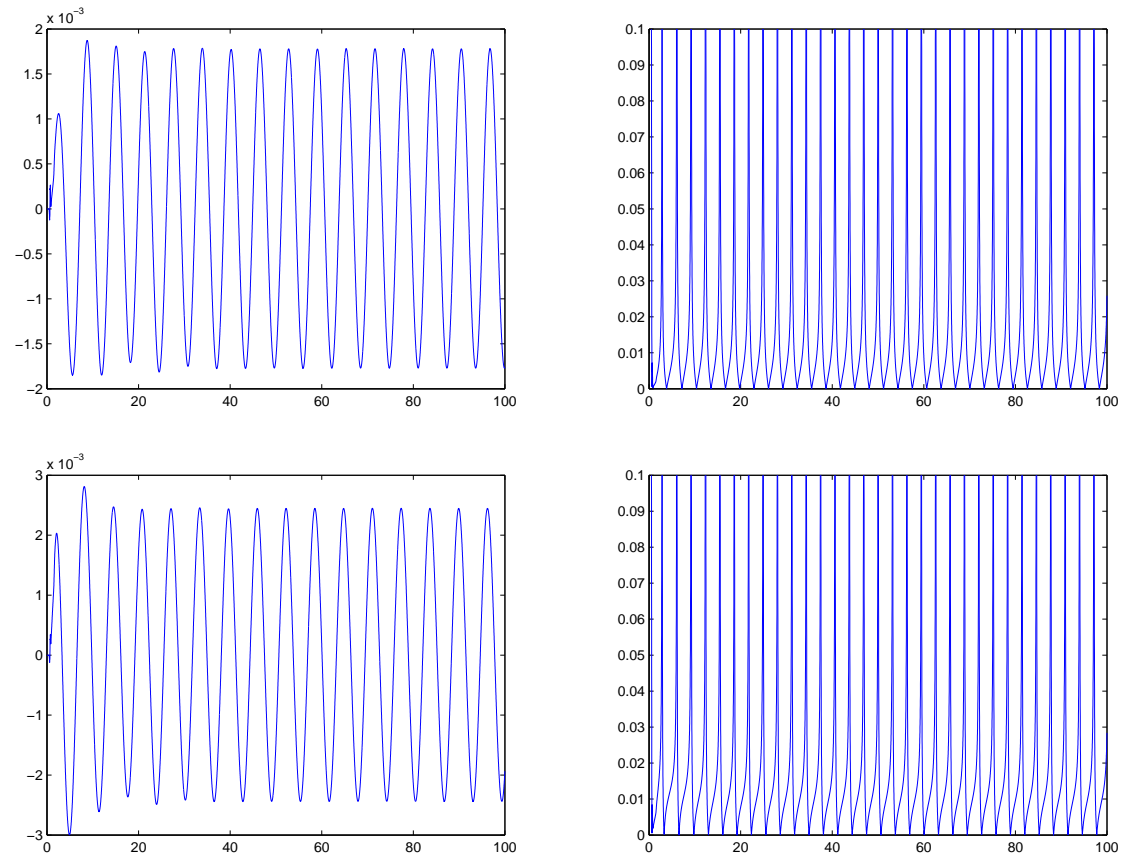

FIG. 6.6. Example 2: The absolute and relative errors of the numerical solution at point $(0.4875,0.4875)$ for different times (top: $d_{1}=d_{2}=0.5$, bottom: $\left.d_{1}=d_{2}=0.25\right)$.

In Figure 6.6, we show the absolute and relative errors of the numerical solution at the above point. It is observed that the long time stability and convergence hold for each choice of the PML parameters.

Finally, we remark that the computational errors in Example 1 increase in time due to the presence of the source for all time and the computational errors oscillate in Example 2 due to the oscillation of the source. They both grow no faster than the polynomial function of $T$ in the error bound in Theorem 5.2. We also remark that the computational errors shown in the numerical examples include both the errors due to the PML method that are analyzed in this paper and the errors of the discretization 
of the PML system which deserve further studies.

Acknowledgement. The authors would like to thank the referee for the constructive comments.

\section{REFERENCES}

[1] S. Abarbanel and D. Gottlieb, A mathematical analysis of the PML method, J. Comput. Phys.134 (1997), 357-363.

[2] D. Apelö, T. Hagstrom and G. Kreiss, Perfectly matched layers for hyperbolic systems: General formulation, well-posedness and stability, SIAM J. Appl. Math. 67 (2006), 1-23.

[3] E. Bécache and P. Joly, On the analysis of Bérenger's perfectly matched layers for Maxwell equations, Math. Modelling Numer. Anal. 36 (2002), 87-119.

[4] E. Bécache, P.G. Petropoulos and D. Gedney, On the long-time behavior of unsplit perfectly matched layers, IEEE Trans. Antennas Propagat. 52 (2004), 1335-1342.

[5] J. P. Bérenger, A perfectly matched layer for the absorption of electromagnetic waves, J. Comput. Phys. 114 (1994), 185-200.

[6] S.X. Chen, Introduction to modern partial differential equations, Science Press, Beijing, 2005 (in Chinese).

[7] Z. Chen, Convergence of the time-domain perfectly matched layer method for acoustic problems, Int. J. Numer. Anal. Modeling 6 (2009), 124-146.

[8] Z. Chen and X. Liu, An adaptive perfectly matched layer technique for time-harmonic scattering problems, SIAM J. Numer. Anal. 43 (2005), 645-671.

[9] Z. Chen and $\mathrm{H}$. Wu, An adaptive finite element method with perfectly matched absorbing layers for the wave scattering by periodic structures, SIAM J. Numer. Anal. 41 (2003), 799-826.

[10] Z. Chen and X. Wu, An adaptive uniaxial perfectly matched layer method for time-harmonic scattering problems, Numer. Math. Theor. Meth. Appl. 1 (2008), 113-137.

[11] Z. Chen and W. Zheng, Convergence of the uniaxial perfectly matched layer method for timeharmonic scattering problems in two-layered media, SIAM J. Numer. Anal. 48 (2010), 2158-2185.

[12] W. C. Chew and W. Weedon, A $3 D$ perfectly matched medium from modified Maxwell's equations with stretched coordinates, Microwave Opt. Tech. Lett. 7 (1994), 599-604.

[13] F. Collino and P. Monk, The perfectly matched layer in curvilinear coordinates, SIAM J. Sci. Comput. 19 (1998), 2061-2090.

[14] A.T. de Hoop, P.M. van den Berg and R.F. Remis, Absorbing boundary conditions and perfectly matched layers - An analytic time-domain performance analysis, IEEE Trans. Mag. 38 (2002), 657-660.

[15] J. Diaz and P. Joly, A time domain analysis of the PML models in acoustics, Comput. Methods Appl. Mech. Engrg. 195 (2006), 3820-3853.

[16] S.D. Gedney, An anisotropic perfectly matched layer absorbing media for the truncation of FDTD latices, IEEE Trans. Antennas Propragat., 44 (1996), 1630-1639.

[17] M.J. Grote and I. Sim, Efficient PML for the wave equation, preprint, University of Basel, 2009.

[18] T. Hagstrom, Radiation boundary conditions for the numerical simulation of the waves, Acta Numerica, 8 (1999), 47-106.

[19] T. Hohage, F. Schmidt and L. Zschiedrich, Solving time-harmonic scattering problems based on the pole condition II: Convergence of the PML method, SIAM J. Math. Anal. 35 (2003), 547-560.

[20] S. Kim and J.E. Pasciak, Analysis of a Cartisian PML approximation to acoustic scattering problems in $\mathbb{R}^{2}$, J. Math. Anal. Appl. 370 (2010), 168-186.

[21] M. Lassas and E. Somersalo, On the existence and convergence of the solution of PML equations, Computing, 60 (1998), 229-241.

[22] W. McLean, Strongly Elliptic Systems and Boundary Integral Equations, Cambridge University Press, Cambridge, 2000.

[23] J.-C. Nedelec, Acoustic and Electromagnetic Equations - Integral Representations for Harmonic Problems, Springer, Heidelberg, 2001.

[24] P.G. Petropoulos, Reflectionless sponge layers as absorbing boundary conditions for the numerical solution of Maxwell equations in rectangular, cylindrical and spherical coordinates, SIAM J. Appl. Math. 60 (2000), 1037-1058.

[25] Z.S. Sacks, D.M. Kingsland, D.M. Lee and J.F. Lee, A perfectly matched layer anisotropic 
absorber for use as an absorbing boundary condition, IEEE Trans. Antennas Propragat. 43 (1995), 1460-1463.

[26] F.L. Teixeira and W.C. Chew, Advances in the theory of perfectly matched layers, In: W.C. Chew et al, (eds.), Fast and Efficient Algorithms in Computational Electromagnetics, 283346, Artech House, Boston, 2001.

[27] E. Turkel and A. Yefet, Absorbing PML boundary layers for wave-like equations, Appl. Numer. Math. 27 (1998), 533-557.

[28] G.N. Watson, A Treatise on The Theory of Bessel Functions, Cambridge, 1922.

[29] L. Zhao and A.C. Cangellaris, A general approach for the development of unsplit field time domain implementations of perfectly matched layers for FDTD grid truncation, IEEE Microwave Guided Wave Lett. 6 (1996), 209-211. 